\title{
Progress in Remotely Triggered Hybrid Nanostructures for Next Generation Brain Cancer Theranostics
}

Nanasaheb D. Thorat ${ }^{1,2}$, Helen Townely ${ }^{3}$, Grace Brennan ${ }^{1}$, Abdul K Parchur ${ }^{4}$, Christophe Silien $^{1}$, Joanna Bauer ${ }^{2}$ and Syed A.M.Tofail ${ }^{1}$

${ }^{1}$ Modelling Simulation and Innovative characterisation (MOSAIC), Bernal Institute, University of Limerick, Limerick, Ireland

${ }^{2}$ Department of Biomedical Engineering, Wroclaw University of Science and Technology, Wroclaw Poland

${ }^{3}$ Nuffield Department of Obstetrics and Gynaecology, Medical Science Division, Oxford University, Oxford, UK

${ }^{4}$ Department of Radiology, Medical College of Wisconsin, USA

\begin{abstract}
:
Progress in nanomedicine has enabled the development of smart hybrid nanosystems for brain cancer theranostics. This novel platform can concurrently diagnose the tumor, start primary treatment, monitor therapy response, and in further extreme cases, initiate secondary treatments. Upon the application of external physical stimulus generally referred as remote stimuli, e.g., alternating magnetic field, visible or near-infrared light, ultrasound radiation, Xray, and radiofrequency, the hybrid nanosystems can release guest molecules/cargoes to the brain tumor. The release of cargoes can be remotely triggered in a controlled manner by physical stimuli. Remote triggering mechanisms include photodynamic therapy, photothermal therapy, phototriggered chemotherapeutics, ultrasound, electrothermal therapy, and magnetothermal therapy. Herein, we review different hybrid nanostructures (HNS) currently used as remotely triggered modalities in brain cancer, such as organic-inorganic HNS, polymer micelles, and liposomes HNS. Moreover, we also summarize the underlying mechanisms that manage such type of remotely triggered brain cancer therapeutics including single- and twophoton triggering, thermoresponsive HNS, photoresponsive HNS, magnetoresponsive HNS, and electrically and ultrasound stimulated HNS. Thus, the review provides a brief/detailed account of current state-of-the-art "smart" HNC-based platforms of novel brain cancer therapeutics. Further the review offers the up-to-date development in this field to neurooncologists, material/nano scientists, and radiologists, so that a rapid clinical impact can be made in this field.
\end{abstract}


Keywords: hybrid nanostructures; nanomedicine; magnetic materials; brain cancer therapy; photothermal therapy

\section{Review Outline:}

1. Introduction: Brain Tumors Current Status

2. Current Approaches v/s Hybrid Nanotheranostics for Brain Cancer

2.1 Remotely Triggered Hybrid Nanostructures

2.2 Remotely Triggered Hybrid Nanostructures: Brain Cancer Theranostics

2.2.1 Why remotely triggered therapies?

\subsubsection{Remotely Triggering Modalities}

3. Remotely Triggering Modalities in Brain Cancer Theranostics

3.1 Light Triggered Hybrid Nanostructures

\subsubsection{Inorganic Hybrid Nanostructures}

3.1.2 Polymers and Liposomes

3.1.3 Strengths and challenges

3.2. Two-Photon Triggered Hybrid Nanostructures

3.3 Magnetically Triggered Hybrid Nanostructures

3.3.1 Organic-Inorganic HNS

3.3.2 Strengths and challenges

3.4 Ultrasound Triggered Hybrid Nanostructures

3.4.1 Liposomes

3.4.2 Organic-Inorganic HNS

3.4.3 Strengths and challenges

3.6 Electro-Magnetically Triggered Hybrid Nanostructures

3.6.1 Strengths and challenges

4. Challenges, Conclusion and Future Outlooks

4.1 Challenges

4.1.1 HNS targeted delivery

4.1.2 HNS surface properties

4.1.3 Other challenges

4.2 Conclusion

4.3 Future Outlooks 


\section{Introduction: Brain Tumors Current Status}

In 2012 , there were 256,213 incidences $(3.4$ people per 100,000$)$ of primary malignant brain and central nervous system (CNS). Cancer therapeutic strategies involving dual targetable UCNPs carrying both photothermal/photodynamic sensitizers was developed for the synergistic treatment of GBM ${ }^{1}$ which has a higher rate of incidence $(5.1$ per 100,000$)$ in developed countries than lesser developed countries $(3.0 \text { per } 100,000)^{2}$. This is also the cancer that is most commonly seen in children under the age of 14 , accounting for $25 \%$ of all tumors in this age range ${ }^{3}$. Glioblastoma (GBM) is considered to be the most common and aggressive form of primary brain tumor with a median survival of less than 2 years after initial diagnosis and maximal therapy ${ }^{4}$. Reappearance of the tumor at the same location where the primary tumor was originally located is the foremost cause of death by brain cancer ${ }^{5}$.

Nearly half of the patients having brain cancer will have metastasis all through the course of their condition. Metastases are particularly difficult to treat because the brain cancer patients are frequently fragile, malnourished, and have an abundance of co-morbidities. Even with the best available treatments, median survival time in metastatic brain cancer patients reduces to less than half of typical survival time ( $\sim 10$ months $)^{6}$. Molecular changes in cancer cells drives the expansion and progression of brain cancer, treatment of which becomes highly difficult due to the complexity of the brain tissues, and the difficulty in passing therapeutic agents.

The human brain is an exceptionally complicated organ with complex anatomical structure and physiological functions. Its functions include perception, learning, memory storage, processing information, arousal, motor control and motivation. It is the master controller and central processing unit of the human body. Brain cancer therapy therefore demands an extremely vigilant and delicate removal of all tumorous tissues without causing damage to the surrounding healthy tissue ${ }^{7}$. This is very challenging to achieve in practice and leaves open the possibility of re-occurrence due to incomplete removal.

The symptoms of brain cancer depend upon the size, position and rate of expansion of the tumor. Common symptoms in patients with a primary brain cancer consist of nausea, vomiting, cognitive changes, personality changes, hemiparesis, aphasia, urinary incontinence, hemineglect (or spatial neglect), visual field deficiency, and apprehension. Headaches are present in about $50 \%$ of individuals at the time of diagnosis, but frequently with a nonspecific 
pain pattern. Headaches that worsen with time or become more frequent, unilateral headaches that gives a constant throbbing sensation on one side of the head in patients older than 50 years are often taken as signs to be a tumor-associated pain rather than just a standard headache ${ }^{8}$.

Brain tumor diagnosis typically starts with imaging by magnetic resonance imaging (MRI) or X-ray computed tomography (CT) to confirm and locate the anatomical site of the tumor. MRI provides superior temporal and spatial resolution along with anatomical details. As such, MRI has largely replaced CT imaging in brain cancer diagnosis. Molecular imaging techniques such as positron emission tomography (PET) are also utilised in brain cancer diagnosis. PET can efficiently characterize brain cancer tumors especially in investigating the metabolic processes such as receptor binding, oxygen metabolism, DNA synthesis, enzyme activity, and blood flow consumed. This technique is beneficial to identify metastasis sites in the brain. However, for most brain tumors the quantification of the actual tumor volume and identification of precise tumor margins remains difficult even with using these state of the art diagnostic imaging techniques ${ }^{9}$. Contrast agents are used to overcome these limitations. Many magnetic and gold based hybrid nanostructures (HNSc) are currently used as excellent contrast agents in MRI, CT and PET imaging (Figure 1).

Brain cancer therapeutics remains challenging, specifically the delivery and release of drugs to the brain tumor site. Due to the presence of tight junctions with of high electrical resistance (1500-2000 $\Omega \mathrm{cm}^{2}$ ) between endothelial cells, which usually excludes most therapeutics ${ }^{10}$. This brain protection mechanism is called the Blood Brain Barrier (BBB). In order to efficiently transport and release therapeutic cargoes into the brain bypassing the BBB, various strategies have been developed. They include chemical alteration of chemotherapeutic drug and prodrugs, temporary disruption of the tight junctions by physical forces, local delivery into the brain by neurosurgery and nanoparticle-mediated delivery ${ }^{11}$. In recent years, remotely triggered strategies combined with HNS have been developed to supress the BBB for brain theranostics. These strategies include focused ultrasound and magnetic field interaction with the $\mathrm{BBB}$ and light triggering of the BBB. Physical triggering techniques are typically noninvasive, allow for good selectivity of opening position, less side effects, and reusable. Hence, physical triggering techniques are advantageous compared with the common methods used in the clinic such as hyperosmotic glucose in opening the $\mathrm{BBB}^{12}$.

In contrast from previous reviews ${ }^{13,14}$, this progress update review report will mainly emphasize the most recent advances of multifunctional hybrid nanostructures for remotely triggered brain cancer therapy with high efficiency and safety by taking advantage of each composition and synergetic therapeutic effect, which have never been reviewed in such a 
systematic way for brain cancer. Chakroun et. al., provides a recent review of various modalities, advantages and limitations of current brain cancer therapies such as surgery, radiotherapy, systemic therapy, localized gene therapy and immunotherapy ${ }^{15}$. Thus, the current review outlines latest advances in detail by the analysis of different physical stimulus, focusing on the unique physical phenomenon of the material, functional property, as well as the therapeutic ability and mechanism for brain tumor cells removal or killing.

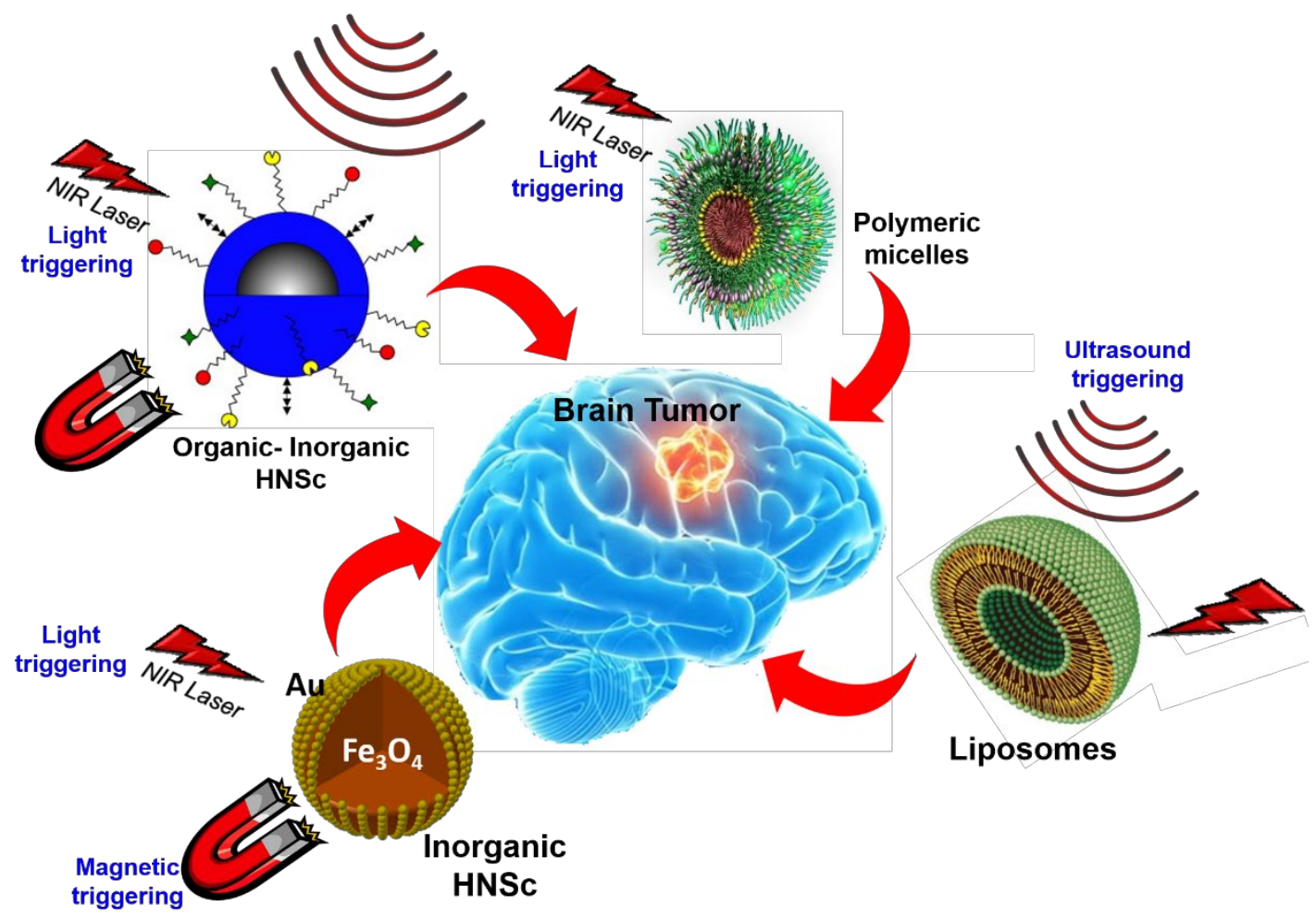

Figure 1. Different types of hybrid nanostructures (HNS) used as remotely triggered modalities in brain cancer theranostics.

\section{Current Approaches v/s Hybrid Nanotheranostics for Brain Cancer}

In addition to the current brain cancer treatments such as surgical excision, radiation therapy (e.g. whole brain radiation therapy; WBRT), and chemotherapy, new treatment methods have also been studied, for example angiogenesis inhibitors therapy ${ }^{16}$, immunotherapy ${ }^{17}$, photodynamic therapy ${ }^{18}$, hyperthermia therapy ${ }^{19}$ and gene therapy ${ }^{20}$.

Nanotechnology has enabled the advancement of smart theranostic systems that can simultaneously diagnose disease, start primary treatment, monitor the response, and then, if necessary, initiate secondary treatments ${ }^{21}$. This field of nanotechnology is generally referred to as "Nanomedicine" or more precisely "Theranostic Nanomedicine." Recently, various 
mono-component nanostructures, e.g. polymer micelles, inorganic-organic core-shell nanostructures, mesoporous silica, liposomes, as well as their hybrid derivatives, have been exploited for brain cancer therapy or in overall nanomedicine based cancer theranostics. Hybrid nanostructures (HNS) have advantages over mono-component nanostructures, as they can possess a synergistic combination of different functions. HNS can also carry diagnostic and therapeutic payloads to the tumor and release it by remote triggering. The advantage of the broad field of nanomedicine in brain cancer therapy over conventional cancer treatments has been reviewed in recent literature ${ }^{7}$. In the rest of the current review, we are covering progress in hybrid nanostructure-based brain cancer therapies and how these cations are controlled under external stimuli such as external magnetic fields, near-infrared (NIR) light, ultrasound etc and advancing brain cancer theranostics (Figure 1).

\subsection{Remotely Triggered Hybrid Nanostructures}

"Smart" nanosystems that carry and release drugs or cargos and in response to an external stimulus induce a toxic effect to the surrounding area are commonly referred to as remotely triggered nanosystems. These systems promise superior clinical results over commonly used conventional systems that release their cargo either passively or are stimulated internally ${ }^{21}$. In the past few decades, various nanovehicles or nanostructures have been developed as imaging contrasts in MRI and CT, ultrasound imaging, targeted drug delivery systems, or as therapies such as photothermal, photodynamic, magnetic hyperthermia. Particularly, if more than one type of nanostructure can be incorporated in a nanoassembly, they are referred to as a "hybrid nanostructures"22,23. Organic - Inorganic hybrids are a type of promising nanomaterials which display remarkable physiochemical and biological properties, which makes them highly attractive in cancer theranostics ${ }^{24,25}$. Hybrid nanostructures can be designed alone or it may be embedded into other biocompatible materials to yield new functionalities. Specially designed organic hybrid nanostructures in brain cancer theranostics can be synthesized from a selection of raw materials, such as polymers, lipids, nucleic acids, and proteins. Inorganic hybrid nanostructures in theranostics can be obtained by combining features of individual properties of other inorganic materials such as silica, iron oxide, carbon, graphene, gold or silver. The biological interactions of these inorganic hybrid nanostructures could be designed and enhanced by surface functionalization, which can facilitate further potential use in pharmaceutical applications ${ }^{25}$. The exciting properties of organic and inorganic nanostructures have inspired scientists to combine them and design new classes of functional hybrid 
nanostructures for advanced cancer therapies. After the activation by a remote stimuli, e.g. alternating external magnetic field, NIR light or ultrasonic radiation, the release of conjugated molecules can be prompted in a measured fashion into the cellular environment.

\subsection{Remotely Triggered Hybrid Nanostructures: Brain Cancer Theranostics}

\subsubsection{Why remotely triggered therapies?}

The treatment of brain tumors is extremely complex and challenging, largely because of the intracranial tumor location ${ }^{26}$. Intracranial tumors are essentially 'shielded' from the effects of most clinically administered foreign agents, due to the blood-brain barrier (BBB). The BBB regulates the brain microenvironment by providing both a physical and metabolic barrier, controlling the access of molecules to both the brain and brain tumors ${ }^{27}$. Thus, the major contribution to the failure of brain cancer therapy is the inadequate passage of therapeutic molecules across the BBB. Therefore, any drug delivery system targeting brain tumors primarily needs to be capable of safely delivering an adequate amount of chemotherapeutic drugs to the brain tumor location, ideally with negligible or zero early release during the in vivo transport to minimise damage to healthy tissues. This latter point can be achieved by the incorporation of specific stimuli for activation ${ }^{28}$. The stimulus may be the in vivo tumor cell microenvironment (e.g change of $\mathrm{pH}$ in a tumor cell), or a remote stimulus such as light, magnetic field, or ultrasound. The use of in vivo stimuli can result in an uncontrolled release of the chemotherapeutic payload. It is anticipated that these drawbacks could potentially be overcome by using HNS in combination with remote stimuli/triggering (Figure 2). Fortunately, these remote triggers have fewer side-effects, are widely accessible and can be easily manipulated for optimal controllability, making them favourable for brain cancer theranostics ${ }^{29}$.

\subsubsection{Remotely Triggering Modalities}

Two of the most commonly used remotely triggering modalities are NIR light and magnetism. The other non-invasive and controlled modality uses a magnetic field with, for example, superparamagnetic nanoparticles which in the absence of an external magnetic field have negligible magnetism but are highly sensitive to external magnetic fields if present. Thus, superparamagnetic nanoparticles can be used as drug carriers under the magnetic field, and can be trigged under externally applied magnetic fields (both alternating current and direct current). High-frequency ultrasound is another effective modality for triggering thermosensitive lipids conjugated to nano-sized liposomes. Upon irradiation from high-frequency ultrasound, these 
liposomes change phase. The currently used physical remotely triggered modalities based on HNS in brain cancer theranostics are summarised in Figure 1 and 2.

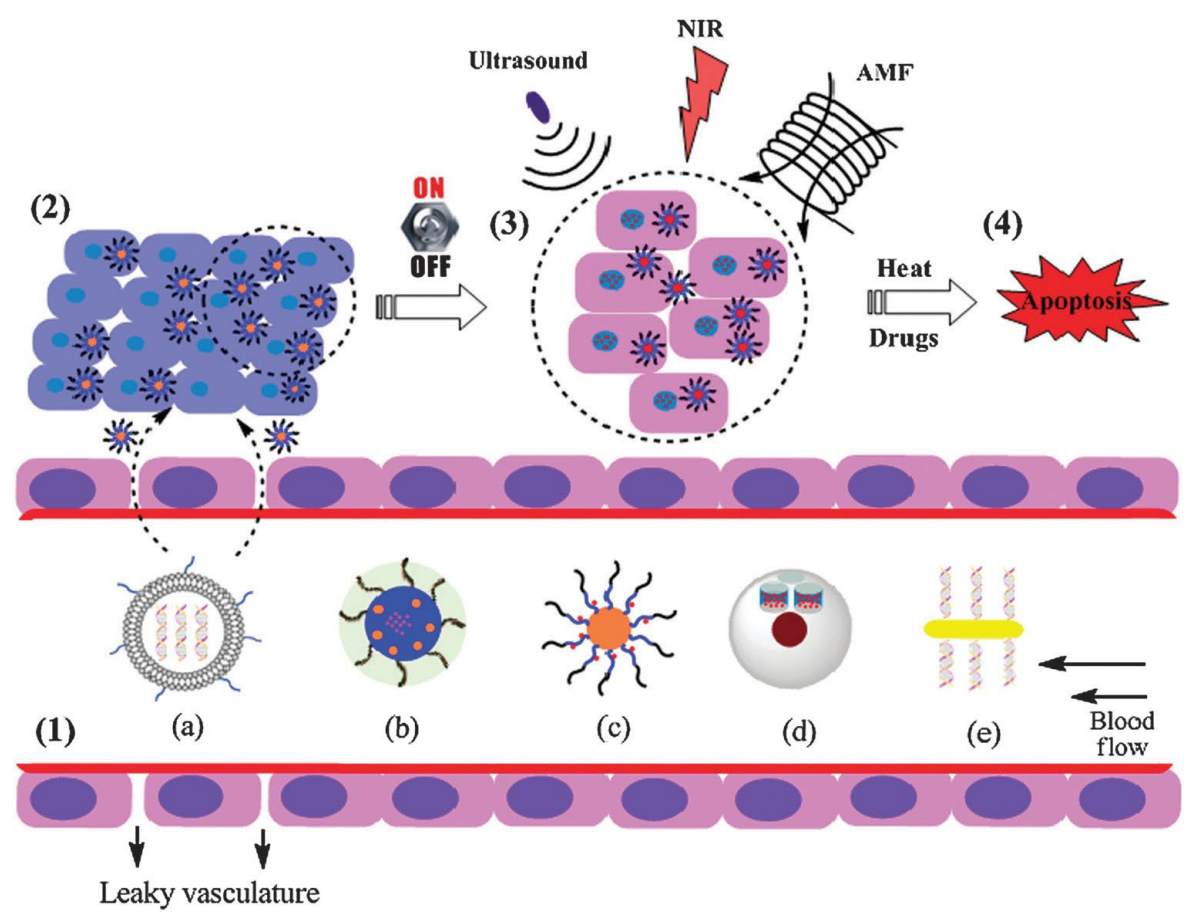

Figure 2. (1) Hybrid nanovehicles with different kinds of structures designed for remotely triggered drug delivery systems, such as liposomes (a), cross-linked polymer nanogels or micelles (b), organic-inorganic core-corona nanohybrids (c), mesoporous silica-based nanovehicles engineered with gating moieties (d) and host-guest nano-conjugates (e); (2) delivery of the guest molecules to desirable tumour sites via passive or active targeting through the vasculature; (3) triggered release of the loaded cargos through remote stimuli, e.g. ultrasound, light (such as near-infrared laser) or alternating magnetic field (AMF); (4) tumour cell apoptosis resulting from the synergistic effect of thermal and chemotherapeutic contributions. Reproduced with permission from ref 30. Copyright 2015 The Royal Society of Chemistry.

\section{Remotely Triggering Modalities in Brain Cancer Theranostics}

\subsection{Light-Triggered Hybrid Nanostructures}

Light is a widely utilised external stimulus, almost all living organisms interact with light in some way, and triggering a biological effect with light is therefore not a new phenomenon. In the human body, the pigment melanin which resides in the epidermal layer of the skin results in skin darkening and thus reduces the skin's ability to produce vitamin D from sunlight ${ }^{31}$. Plants can interact with sunlight by small pigments named chlorophyll, which captures light energy and the energy is then transferred, stored, utilized, and dissipated ${ }^{32}$. In the field of 
theranostics, more applications use the NIR spectrum rather than the UV/visible as it can penetrate to a greater depth in tissues due to the minimal scattering, or autofluorescence, of biological species in this region of the light spectrum. Many HNS interact with NIR light and this can be remotely triggered to exert antitumor activity through light irradiation. The advantage of light activated cancer theranostics has been reviewed in recent literature by Kim et.al. ${ }^{33}$.

\subsubsection{Inorganic Hybrid Nanostructures}

Light interaction with inorganic noble metal-based HNS can produce heat or by radiating its energy as light through the strong surface plasmon oscillation/resonance phenomenon. This interesting property allowing the application of these HNS in photodynamic therapy (PDT), photothermal therapy (PTT), and optical imaging of brain tumors ${ }^{34}$. Gold based HNS with strong NIR absorption are ideally suited for PTT of brain tumors. Gold nanoparticles with particular geometry (nanorods, nanostars, etc.), for example, exhibit a surface plasmon resonance (SPR) in the NIR region, and are therefore sensitive to NIR light ${ }^{35}$. A variety of gold HNS have been investigated as the remotely triggered PTT mediator in brain cancer theranostics ${ }^{36}$. Hybrid Gold nanoparticles conjugated with various polymers, biomolecules, silica and other inorganic moieties are widely studied for biosensing and molecular diagnostic applications $^{37}$ as well as remotely triggered brain cancer theranostics. A recent review by Hirschberg et al., summarized all hybrid gold nanostructures for brain theranostics ${ }^{38}$. Gao et al., developed hybrid gold nanoprobes that can penetrate the blood-brain barrier to reach the brain tumor $\operatorname{sit}^{39}$. These nanoprobes show high performance magnetic resonance (MR) and surface-enhanced Raman spectroscopy (SERS) signals, which further improve the brain tumor surgical outcome by providing high specificity, safety, quantification and aids in the reduction of image distortion caused by inevitable brain shifts during surgery. Meyers et.al., developed Au nanoparticles (NPs) loaded with the PDT drug Silicon phthalocyanine (Pc 4) as a lighttriggered $\mathrm{HNC}$ for brain tumor ${ }^{40}$. By utilising Epidermal Growth Factor (EGF) peptidemodified Au NPs and the PDT drug in combination, they were able to effectively target and deliver therapy to subcutaneous brain tumor-bearing mice. Dixit et.al., synthesized Au NPs conjugated to a transferrin peptide $\left(\mathrm{Tf}_{\mathrm{pep}}\right)$ and $\mathrm{Pc} 4\left(\mathrm{Tf}_{\mathrm{pep}}-\mathrm{Au}-\mathrm{Pc} 4\right)$ and successfully using them in photo-triggered orthotopic brain tumors. ${ }^{41}$ Another very novel application uses silica-coated gold nanorods which mimic the rabies virus (RVG-PEG-AuNRs@SiO $\mathrm{Si}_{2}{ }^{42}$. The hybrid gold nanorods (AuNRs) was functionalised with RVG29, which facilitated their transport across the 
BBB and target the brain by way of the CNS. In addition, the RVG-PEG-AuNRs@ $\mathrm{SiO}_{2}$ when externally excited by a NIR laser ( $808 \mathrm{~nm})$ irradiation and effectively suppressed brain tumors in mice (Figure 3).

In addition to gold, rare earth elements have been used as promising candidate in lighttriggered brain cancer theranostics such as $\mathrm{Tb}^{3+}-\mathrm{Au}$ nanostars ${ }^{43}$. Yuan et.al., developed highly plasmon-active gold nanostars for a pulsed laser-modulated plasmon-enhanced therapeutic system for brain tumor microvascular permeabilization. This system is a truly organicinorganic hybrid, which combines liposomes and magnetic plasmonic NPs for a trimodality image-guided drug delivery which has been shown effective for brain cancer theranostics ${ }^{44}$. This system can overcome the drawbacks of a single gold-magnetic or organic hybrid system and can target payloads effectively to the brain under light and magnetic field guidance. Additionally, hybrid silica-gold nanoshells have been shown to be effective in treating brain tumors under laser light. ${ }^{45}$ In recent report $\mathrm{Fe} @ \mathrm{Fe}_{3} \mathrm{O}_{4} \mathrm{NPs}$ have been modified with ${ }^{125} \mathrm{I}-$ c(RGDyK) peptide and PEGylated ( ${ }^{125}$ I-RGD-PEG-MNPs) to allow photo-triggered MRI and single-photon emission computed tomography (SPECT) for image-guided brain tumor theranostics ${ }^{46}$. This $\sim 40 \mathrm{~nm}$ HNS exhibited good tumor targeting ability, low mononuclear phagocyte uptake and has achieved photo-triggered cargo release along with the photothermal effect that can be monitored under MR imaging on the $\alpha_{\mathrm{v}} \beta_{3}$-positive U87MG glioblastoma xenograft model. Doped $\mathrm{LaF}_{3}$ NPs in combination with a photosensitizer have also been developed for light-triggered brain cancer therapeutics ${ }^{47}$. This novel HNC combines lighttriggered non-invasive photo-therapy under soft X-ray $(180 \mathrm{kVp})$ and as a proof of concept activated the photosensitizer, mesotetra(4-carboxyphenyl)porphyrin (MTCP).

To achieve the multifunctionality in brain cancer theranostics organic dye/polymer conjugated silica HNS are usually proposed. These HNS are stable both physicochemically and biochemically, and are inherently immune to hydrolysis and enzymatic degradation ${ }^{48}$. Extensive meso/nano porosity and pore volumes enable these HNS to hold extraordinarily large loadings of chemotherapuetic cargo to enhance drug treatment efficacy while avoiding its side effects. Benachour et.al., recently demonstrated light stimulated multimodal brain cancer therapy by using surface-localized tumor vasculature targeting NRP-1 grafted gadolinium chelate conjugated silica nanostructures ${ }^{49}$. These multifunctional HNS achieved efficient photodynamic activity on orthotopic human brain tumor (U87) along with MRI. Tang et.al., proposed a new type of dynamic nanoplatform (DNP) based on methylene blue conjugated silica $\mathrm{HNS}$, that only delivers ${ }^{1} \mathrm{O}_{2}$ under photodynamic triggering for brain cancer therapy ${ }^{50}$. 
Since the discovery of helical microtubules of graphitic carbon called carbon nanotubes (CNTs) in $1991^{51}$, CNTs have attracted increasing attention for their versatile applications in brain cancer theranostics ${ }^{52}$. The hollow tubular structure of CNT is advantageous to attach various biomolecules, drugs and targeting moieties. This feature of CNT is elaborated in a recent report, CNT's were functionalised with an immune adjuvant $\mathrm{CpG}$ oligodeoxynucleotides $(\mathrm{CpG})$, fluorescently labeled with Cy5.5 and were intratumorally injected into an intracranial GL261 glioma for antiglioma effect ${ }^{53}$. Eldridge et.al., used a dense coating of phospholipid-poly(ethylene glycol) on multiwalled CNTs (MWCNTS) allowing for better diffusion through brain phantoms ${ }^{54}$. These HNS were also exposed to NIR light as a novel therapy for non-resectable brain tumors and tumors that are resistant to current therapeutic modalities. By taking advantage of the ultrahigh loading ability of molecules through $\pi-\pi$ stacking, nano-sized graphene oxide (GO) has been used as a multifunctional HNS in cancer therapy ${ }^{55}$. Porphyrin functionalized-GO has been used to facilitate photothermal ablation of glioblastoma cell line U87-MG under $808 \mathrm{~nm}$ irradiation, and further validated by in vivo experiments where the tumor was completely eliminated ${ }^{56}$. In the recent review article from Cardano et.al., photo-responsive graphene and carbon nanotubes for biological systems including brain cancer was reviewed ${ }^{57}$. Systemic toxicity, long-term toxicity, and dosedependent toxicity of CNT's and GO towards healthy brain cells still requires study to enable further clinical translation.

The light stimulation of HNS at further depths within solid tumors such as brain tumors is often impaired by the limited tissue penetration depth of ultraviolet or visible light (typically less than $2 \mathrm{~mm})^{58}$. Hence, to improve the therapeutic efficiency in intracranial tumors lanthanide doped hybrid upconversion nanoparticles (UCNPs) have been employed in recent studies. In pioneering work by Tsai et.al., therapeutic strategy involving dual targetable UCNPs carrying both photothermal/photodynamic sensitizers was developed for the synergistic treatment of malignant brain cancer such as glioblastoma multiforme (GBM) tumor ${ }^{59}$. UCNPs conjugated with PEG/angiopep-2 enable GBM-specific co-delivery of the photothermal agent (IR-780), and photodynamic sensitizer (5,10,15,20-tetrakis(3-hydroxyphenyl) chlorin (mTHPC)) which enables external light-triggered targeted therapy. In another study Ni et.al., developed multifunctional dual-targeting imaging nanoprobes for the penetration of the BBB and the application of intracranial light-activated glioblastoma theranostics ${ }^{60}$. PEGylated Gddoped UCNPs were designed to achieve systematic in vitro and in vivo MR/NIR-to-NIR upconversion luminescence imaging of intracranial glioblastoma, which can potentially be 
further expanded into phototherapy. Very recently novel hybrid blue-emitting $\mathrm{NaYF}_{4}: \mathrm{Yb} / \mathrm{Tm} @ \mathrm{SiO}_{2}$ UCNPs are developed for brain stimulation under NIR light. This novel HNS are can upconvert near-infrared light from outside the brain into the local emission of blue light, thus providing new horizon in light triggered brain cancer therapy in near future.
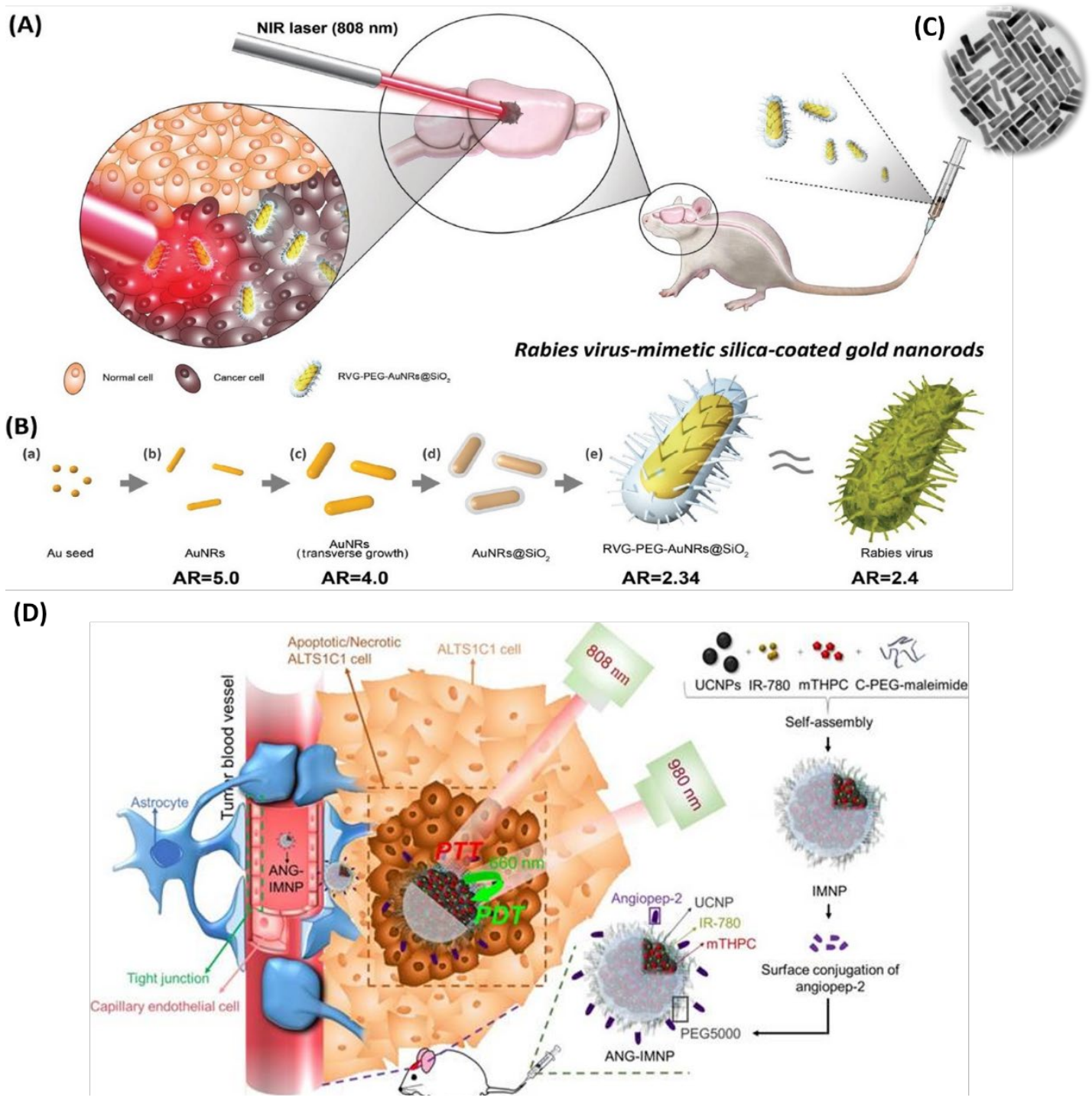

Figure 3. Schematics of recently used different light triggered HNS for brain cancer theranostics. (A) A scheme showing brain delivery of the rabies virus-mimetic RVG-PEGAuNRs@SiO2 via neuronal pathway and photothermal therapy using NIR laser. (B) A scheme of the synthesis strategy for fabricating rabies virus-mimetic silica-coated gold nanorods (RVG-PEG-AuNRs@SiO2), (C) AuNRs (after transverse growth). Panels A-C reproduced with permission from ref 42. Copyright 2007 WILEY-VCH Verlag GmbH \& Co. KGaA, Weinheim. (D) Illustration of active BBB penetration and the photothermal/photodynamic therapeutic design of hybrid ANG-IMNPs in an orthotopic glioblastoma tumor model. Reproduced from ref 59, under the terms of the Creative Commons Attribution (CC BY-NC) license. Copyright 2018 Ivyspring International Publisher. 


\subsubsection{Polymers and Liposomes}

Organic polymeric nanostructures have been shown to be more biocompatible than inorganic HNS, as they do not contain heavy metals and hence attract attention for brain cancer theranostics. Recently, Jiang et. al., synthesized a new class of semiconducting polymer HNS for brain cancer photoacoustic (PA) imaging. This HNC absorbs light in the NIR-I and NIR-II regions, allowing comparative PA imaging between imaging at $750 \mathrm{~nm}$ and $1064 \mathrm{~nm}^{61}$. Multifunctional polymeric HNS have been developed, containing polylactide-co-glycolide (PLGA) nanoparticles which have the ability to concurrently deliver the dye indocyanine green (ICG) and the chemotherapy agent docetaxel (DTX) to the brain. Through surface functionalisation with the brain-targeting peptide angiopep-2, they achieved combined chemophototherapy for glioma through NIR imaging ${ }^{62}$. Along with NIR image-guided chemophototherapy, these HNS could cause cell death of U87MG cells in vitro, and additionally when studied in vivo, facilitated the prolongation of lifespan of brain orthotopic U87MG glioma xenograft-bearing mice (Figure 4).
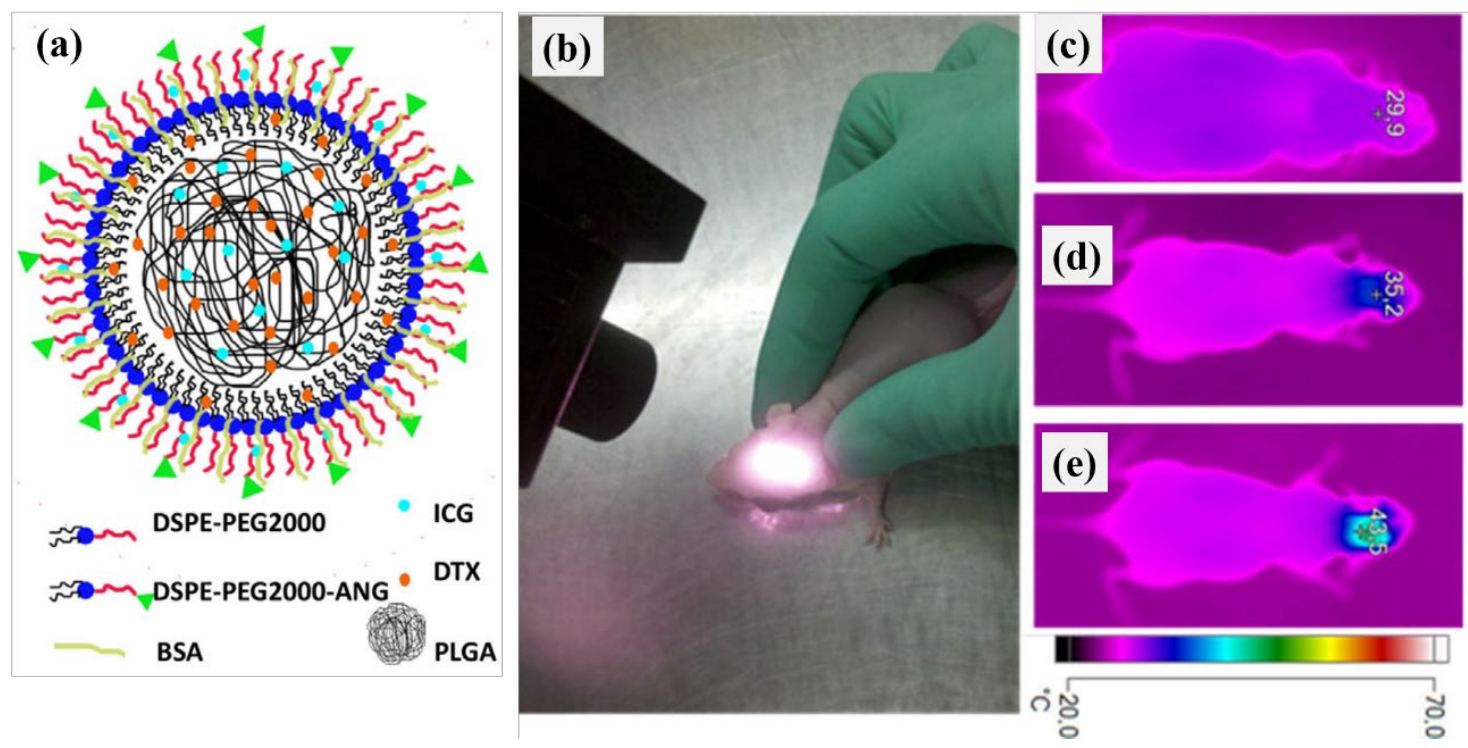

Figure 4. (a) Schematic illustration of the hybrid ANG/PLGA/DTX/ICG NPs structure; (b) The process of irradiation treatment with ANG/PLGA/DTX/ICG, the surface temperature of U87MGglioma-bearing mice at different times (c: $0 \mathrm{~s}$; d: $30 \mathrm{~s}$; e: $60 \mathrm{~s}$.) under an $808 \mathrm{~nm}$ NIR laser irradiation. Reproduced with permission from ref 62. Copyright 2015 WILEY-VCH Verlag GmbH.

Photofrin, a mixture of hydrophobic dimers and oligomers which are primarily linked by ether bonds encapsulated into liposome carriers, have been tested against the U87 glioma 
cell line ${ }^{63}$. These photo-responsive hybrid liposomes effectively enhanced the sensitivity to PDT. In another light triggered PDT study with photofrin, it was shown that there is an increased efficiency against a human glioma which had been implanted into rat brain when compared to photofrin which wasn't encapsulated into a liposome ${ }^{64}$. Recently Chen.et.al., has developed strong NIR activated $\mathrm{H}_{2} \mathrm{O}_{2}$-responsive hybrid liposomes loaded with HRP using 2,2'-azino-bis(3-ethylbenzothiazoline-6-sulfonic acid) (ABTS) as the substrate, (Lipo@HRP\&ABTS) for tumor-specific photothermal therapy in brain cancer theranostics ${ }^{65}$. In the current scenario, liposomes are less studied for brain cancer theranostics, however, in the near future, light activated brain tumor theranostics using a local photosensitizer encapsulated with a liposome may find clinical application.

\subsubsection{Strengths and Limitations}

For the light triggered hybrid drug carries for brain theranostics, the first advantage is the enhanced special therapy in the tumor site and decreased damage of the anticancer drugs to normal brain and neural cells. Light activated brain cancer theranostics are normally sensitive to the radiations of ultraviolet (UV), visible (Vis) and near infrared (NIR) lights. NIR light with wavelengths of $700 \sim 1000 \mathrm{~nm}$, can penetrate up to $10 \mathrm{~cm}$ into living tissue ${ }^{66}$ and normally has no harm to normal brain tissues or neural cells and hence light activated nanomedicine is under intensive research for brain cancer treatment. Because the low-energy of NIR light and high penetration depth, NIR light-triggered cancer therapy has been widely explored for brain cancer.

Meanwhile, this light activated remote stimulation is heavily dependent on the materials properties and therapeutic agents. Both the clinical neuro oncology and exploratory clinical studies demonstrated that the therapeutic effect of single light activated monotherapy is incapable of eliminating the whole brain tumor tissues. The light activated HNS are currently effective only on or just suitable for primary brain tumors. Preventing cancer metastasis effectively is the most challenging task in neuro oncology, currently. Due to the diversity, complexity and heterogeneity of brain tumors as well as the self-limiting of single therapy, light triggering of HNS limiting its application window. Therefore, in order to enhance therapeutic efficacy, the current trend in designing HNS and amplifying therapeutic potential by combining different physical modalities are necessary. 


\subsection{Two-Photon Triggered Hybrid Nanostructures}

While there has been progress with using NIR light, the penetration depth of photons into tissue in this region or the visible region is not deep enough, thus making single-photon HNS remote triggering less effective for brain cancer. Two-photon absorption (TPA)-induced excitation of HNS (photosensitizers) is a more promising approach to increase the penetration of light into tissue. The development of TPA HNS can be achieved by the conjugation of dye molecules onto the nanostructure. The dye molecules simultaneously absorb two photons of lower energy to an excited electronic state (Figure 5a); these excited molecules react with oxygen to generate singlet oxygen which can be used to induce cell death. When two-photon dyes are excited in the near-infrared, light can penetrate deeper into tissue as between $800 \mathrm{~nm}$ and $1100 \mathrm{~nm}$, there is minimal absorption and scattering of light. For an excellent review of the basic physics and chemistry behind the light triggered TPA nanosystems, see Shen et $\mathrm{al}^{67}$. De Gao et al. published the design of 5,10,15,20-tetrakis(1- methyl 4-pyridino)porphyrin tetra(ptoluenesulfonate) (TMPyP), which was encapsulated in a polyacrylamide-based nanoparticle as a TPA modality for brain cancer ${ }^{68}$. Silica modified with organics (ORMOSIL) HNS encapsulated with either PpIX (protoporphyrin IX) photosensitizers or IR-820 NIR fluorophores was synthesized as a TPA mediator as well as a brain cancer mapping agent for brain cancer ${ }^{69}$. Alifu et.al., synthesized the dye called 2-(4- bromophenyl)-3-(4-(4(diphenylamino)styryl)phenyl)fumaronitrile (TPABDFN), which had a broadband two-photon absorption cross-section and strong NIR emission, the dye was then encapsulated in poly(styreneco-maleic anhydride) (PSMA), forming hybrid fluorescent nanostructures (Figure 5b). Subsequently, these HNS were intravenously injected into a mouse, and the threedimensional structure of the blood vessels in the mouse brain could be visualised ${ }^{70}$. This assembly could also be used for two-photon triggered brain cancer therapy. 
(a)

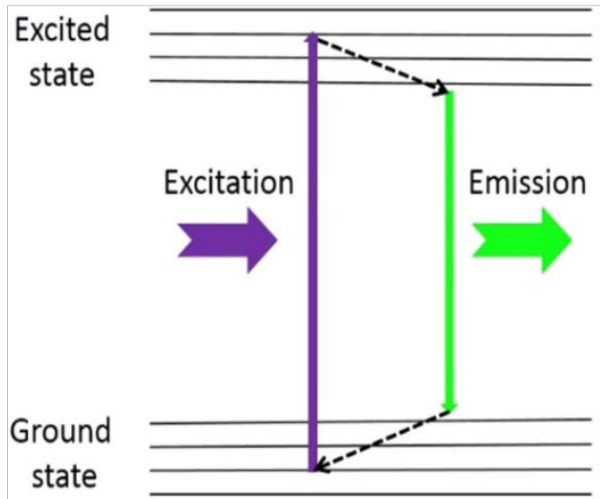

(b)

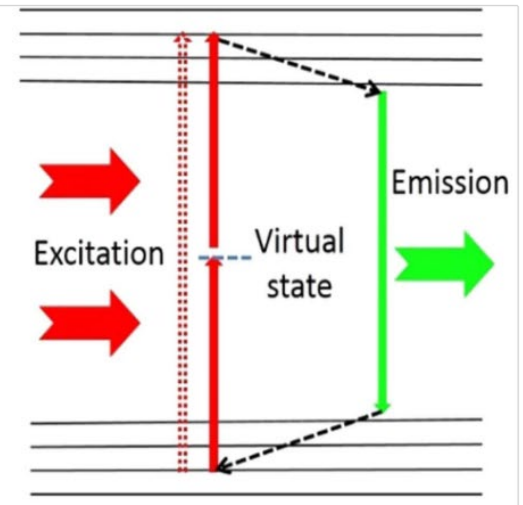

Two-photon excitation

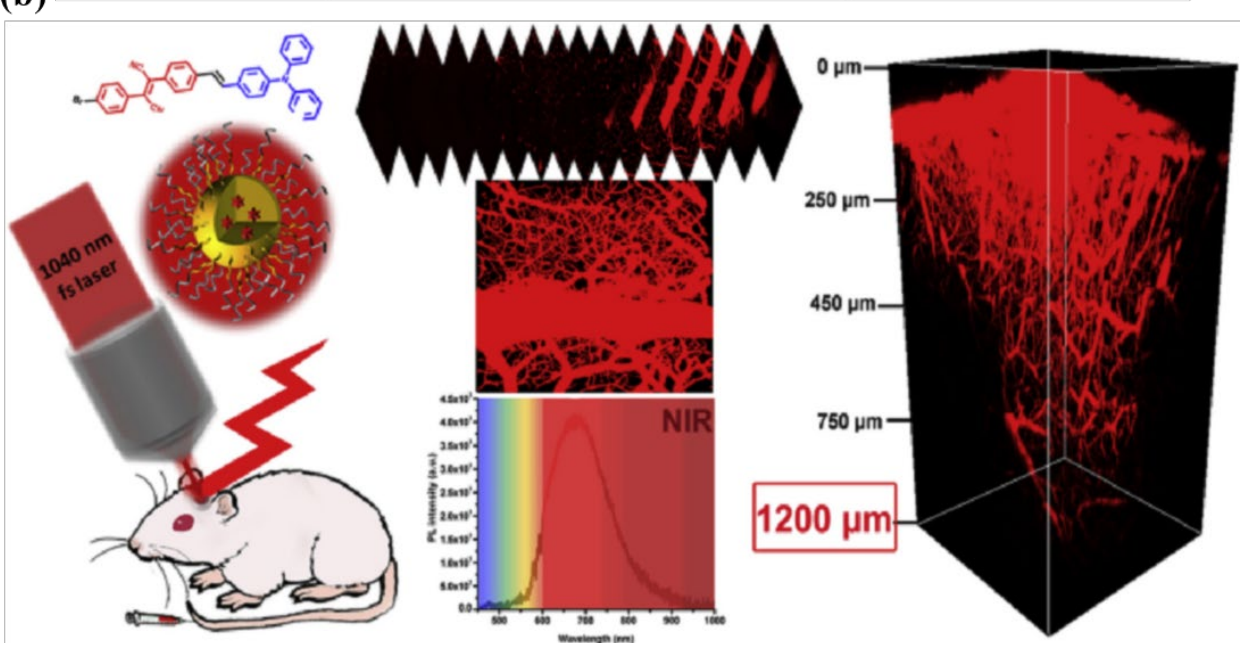

Figure 5. (a) Energy diagrams showing one-photon absorption (OPA), two-photon absorption (TPA). Panel A reproduced with permission from ref 71. Copyright 2017 IOP Publishing Ltd. (b) NIR emissive TPABDFN-PSMA-PEG nanoparticles with large twophoton absorption cross-section were intravenously injected into a mouse, and threedimensional architecture of the blood vessels in the mouse brain was reconstructed vividly with two-photon fluorescence microscopy at imaging depth $\sim 1200 \mu \mathrm{m}$. Reproduced with permission from ref 70. Copyright 2017 Elsevier.

The use of two-photon absorption of NIR light instead of a single visible photon to sensitize HNS or photosensitizers could provide better spatial selectivity and deeper tissue penetration of the brain. Therefore, brain tumors, brain metastasis and angiogenesis can be visualised superiorly than that of one photon excitation by visible light, thus it is a highly promising modality ${ }^{72}$.

\subsection{Magnetically Triggered Hybrid Nanostructures}

Magnetic-responsive HNS or drug carriers enable the remote, on-demand release of chemotherapeutics in brain tumor, which reducing the side effects possible in conventional brain cancer treatments. Magnetic triggering of HNS has many advantages over other physical 
stimulus, as magnetic field penetrates deep into the tissue, and it can stimulate deep tumors by oscillations or heat (magnetic hyperthermia) without damaging normal hypodermal tissues, the basic physics and the targeting mechanism is illustrated in Figure 6a and b. Magnetic field allows a deeper penetration in the brain compared with NIR light or ultrasound (US), and a less harmful ionizing effect compared with X-ray radiations. Adjusting the field strength $(\mathrm{H})$ and frequency (f) of the alternating magnetic field (ACMF) allows for easy regulation of the tumor temperature and drug release ${ }^{73}$, recently termed as magneto-chemotherapy ${ }^{28}$. HNS based magnetically triggered carriers have specific advantages due to their multimodality, and the particles' magnetic controllability also allows active targeting to the brain tumor site, so that it may be possible to avoid the severe side effects of systemic chemotherapy ${ }^{74}$.
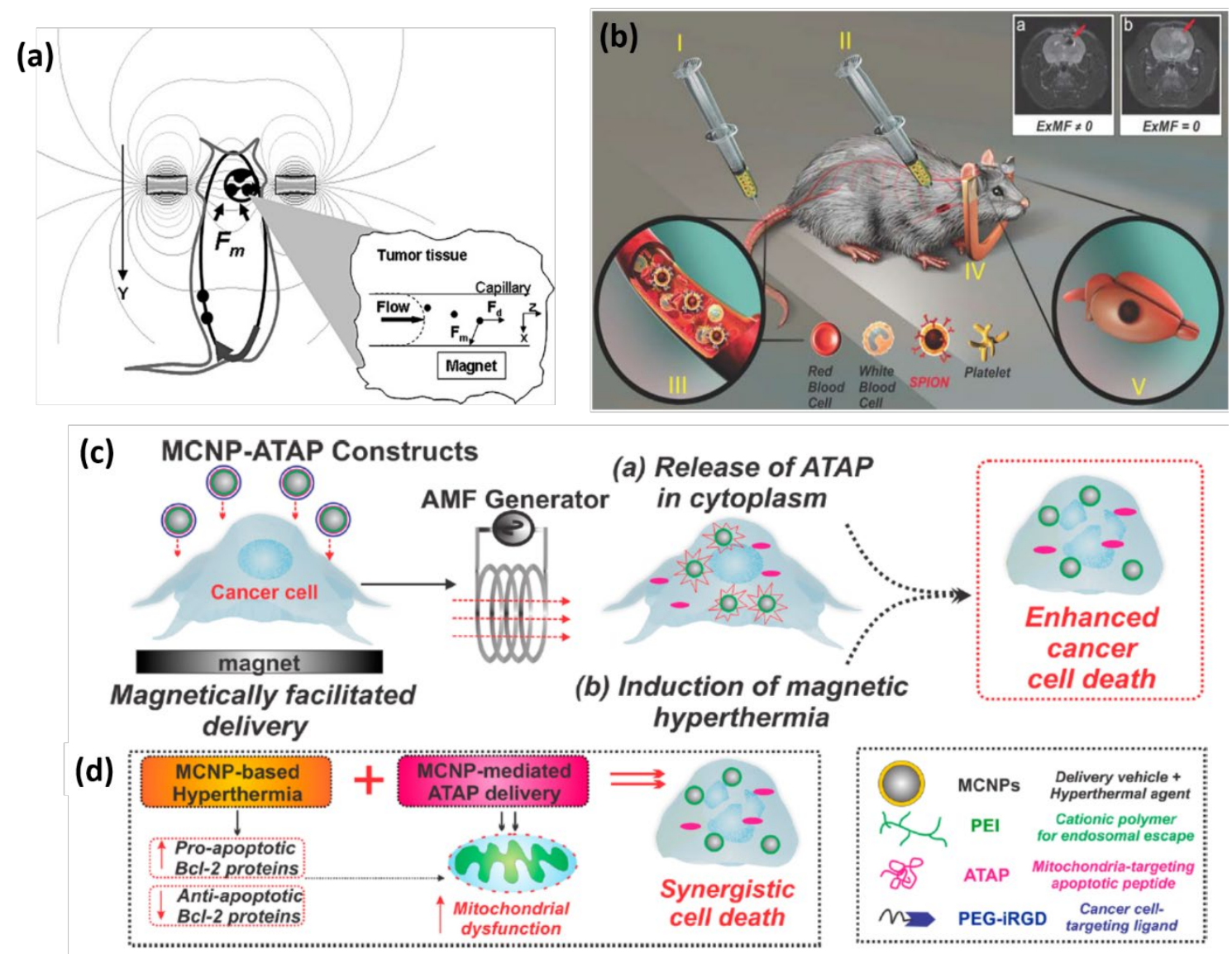

Figure 6 (a) Schematic illustration of brain tumor magnetic targeting following the systemic administration of magnetic nanoparticles. Panel A reproduced with permission from ref 75 . Copyright 2007 Elsevier. (b) Representation of in vivo magnetic targeting using superparamagnetic iron oxide nanoparticles (SPION). The process begins with SPION being administered caudally (I) or by carotid artery (II) and being transported by the blood flow (III). An external magnetic field (ExMF) is applied to target the tumor with SPION (IV), which are concentrated in the tumor tissue (V). MRI showing SPION accumulation (arrow) in tumor with and without ExMF. Panel B reproduced with permission from ref 76. 
Copyright 2017 Walter de Gruyter GmbH. (c) Schematic diagram depicting magnetically facilitated targeted delivery of MCNP-ATAP to brain and breast cancer cells, wherein the release of ATAP from the MCNPs in the cytoplasm coupled with induction of magnetic hyperthermia in the presence of an alternating magnetic field (AMF) can result in synergistic cell death. (d) MCNP-based hyperthermia can result in inactivation of anti-apoptotic bcl-2 proteins, with a concomitant increase in the pro-apoptotic bcl-2 proteins, thus sensitizing the cancer cells toward apoptosis. Panels $\mathrm{c}$ and $\mathrm{d}$ reproduced with permission from ref 77 Copyright 2014 American Chemical Society .

\subsubsection{Organic-Inorganic HNS}

Drug-loaded organic-inorganic magnetic HNS have been shown to be activated in the brain after the application of an external magnetic field ${ }^{78}$. Hybrid nanoparticles comprising of an iron oxide core with a starch coating (designated G100), for example, was developed to penetrate the $\mathrm{BBB}$ and deliver magnetic particles to the brain $^{79}$. The particles were administered intravenously, and magnetic targeting gave a staggering 5-fold increase in the total glioma exposure to MNPs when compared to non-targeted tumors and additionally, a 3.6fold enhancement in the targeting ability of NPs in glioma tissue over normal brain parenchyma. Another example of magnetically triggered brain therapy used core-shell nanocapsules stabilized with a polymer polyvinyl alcohol (PVA)/Polyacrylic acid (PAA) shell and Iron Oxide NPs as the core ${ }^{80}$. This facilitated the simultaneous encapsulation of hydrophilic DOX and hydrophobic Curcumin with great efficiency and resulted in a high amount of local accumulation and cell internalization of the particles both in vitro and in vivo under an external magnetic field.

In addition to localization using a magnetic field, magnetically triggered hyperthermia therapy can have direct therapeutic effects on brain cancer cells, enhance drug delivery and can enable controlled heating of brain tumor tissue in a single modality ${ }^{81}$. Magnetic iron oxide nanoparticles (IONPs) have also been PEGylated and used for remote brain cancer cell killing through magnetic hyperthermia ${ }^{82}$. This study highlights the superior efficiency of multicore (nanoflowers) in comparison to monocore (nanospheres) IONPs for remotely triggered drug delivery and magnetic hyperthermia, resulting in $80 \%$ brain cancer cell death in medically translatable conditions. Prof. Andreas Jordan and his group recently translated the HNS based magnetically triggered hyperthermia to the clinic $^{83}$. The group developed intra-tumoral thermotherapy using IONPs combined with external beam radiotherapy. The primary clinical investigation succeeded following diagnosis of first brain tumor recurrence (OS-2), whereas

the secondary endpoint investigation achieved overall survival after the diagnosis of the primary tumor (OS-1). 
Other HNS composing superparamagnetic iron oxide core NPs have been created with a multifunctional shell consisting of PEG/PEI/polysorbate80 (Ps 80$)^{84}$. These multifunctional HNS were used to encapsulate DOX and it was shown that the cellular uptake of these DOX@Ps 80-SPIONs (superparamagnetic iron oxide nanoparticles) by glioma cells while under a magnetic field was considerably enhanced over that of free DOX in solution. Iron oxide nanoparticles doped with zinc $\left(\mathrm{ZnFe}_{2} \mathrm{O}_{4}\right)$ have also been conjugated with microRNA (miRNA) and used as seed mediators for magnetic hyperthermia therapy (Figure 6c\&d), simultaneously targeting the brain cancer cells and knocking out downstream heat shock proteins (HSP) that would otherwise promote cell survival and inhibit apoptosis following treatment ${ }^{85}$. The same research group further developed hybrid magnetic core-shell NPs for the delivery of a proapoptotic amphipathic tail-anchoring peptide (ATAP) which target mitochondria for both malignant brain and metastatic breast cancer cells ${ }^{77}$.

The development of magnetically triggered non-invasive, tumor-selective delivery system has also been applied to the problem of passing through the $\mathrm{BBB}^{[66,67]}$. The model protein $\beta$-Galactosidase was cationized and tested in orthotopic-glioma-bearing rats. Through using MRI-guided magnetic targeting methodology, physiological arterial hydrodynamics could be preserved during nanocarrier administration and embolization-free and tumortargeted delivery of protein embedded nanocarriers to brain tumor lesions. Magnetically triggered delivery of neural stem cells (NSCs) has also been shown to be a promising strategy for delivering therapeutics to malignant glioma ${ }^{88}$. Spinning disk (SD) magnetic particles were internalized into NSCs and a magnetic field was applied externally to trigger the release of the SD particles, allowing the particles to be uptaken by glioma cells. This method resulted in 50\% glioma cell death. This novel delivery platform has a benefit, the particles do not have to have functional ligands on the surface and consequently are not affected by proteins that may electrostatically interact with the surface.

Combination organic-inorganic HNS has been developed comprising of magnetic fluid-loaded liposomes $(\mathrm{MFLs})^{89}$. The submicronic magnetoliposomes consist of superparamagnetic maghemite nanocrystals which are PEGylated and functionalised with phospholipid vesicles which are rhodamine labelled magnetically guided magnetoliposomes show selective brain tumor targeting and are traceable by MRI, and could, therefore, prevent damage to healthy brain tissue during treatment. Magnetosomes, which are naturally synthesized by magnetotactic bacteria have been loaded with poly-1-lysine to yield a stable and non-pyrogenic HNS suspension for triggered brain cancer theranostics ${ }^{90}$. Magnetic $\mathrm{Fe}_{3} \mathrm{O}_{4}$ cores 
synthesized by magnetotactic bacteria and coated with the polymer poly[aniline-co- $N$-(1-onebutyric acid) aniline] (SPAnH) with a size distribution of $89.2 \pm 8.5 \mathrm{~nm}$ was reported for use in magnetically triggered brain cancer theranostics.

An innovative combined imaging/manipulation platform for successful translation of magnetic HNS in the brain cancer theranostics was developed by I. Weinberg et.al ${ }^{91}$. The platform allowed MNPs to be driven and concentrated to the desired target in the brain to reduce chemotherapy side-effects. E. Alphandéry et.al., introduced a new type of hybrid magnetosomes chains, which have enhanced properties in comparison to commonly used chemically synthesized $\mathrm{HNS}^{92}$. AMF applications exhibited either full brain tumor disappearance in $40 \%$ of mice or no tumor regression using magnetosomes treated with novel hybrid magnetosomes chains.

\subsubsection{Strengths and Challenges}

Combining magnetic triggering and chemotherapy along with magnetic hyperthermia may overcome the problems in the individual brain cancer treatments. These multimodal magnetically triggered nanomedicine approach can cure brain cancer by producing different synergistic effects such as: brain tumor tissues shrinking by magnetic hyperthermia which will further increases the intra and extracellular space to allow chemotherapeutic drugs to spread throughout the tumor and destroy the tumor tissues in the entire tumor. The other strength of the magnetic remote triggering of HNS is the utilization of gradual release of anticancer drugs from the HNS under ACMF, which prevents the anticancer drugs from escaping the tumor and reduces side effects. The HNS based magnetic hyperthermia combined with drug release can potentiate the effect of the anticancer activity within tumor and enhancing the destruction of entire tumor without affecting surrounding environment ${ }^{93}$.

The main challenge in magnetically triggered theranostics is controlling the thermal dose due to high amplitude ACMF, as the local and uncontrolled overheating during magnetochemotherapy may induce surrounding normal brain tissue necrosis. Self-regulated hybrid nanoheaters with ability of magnetic phase change to regulate critical temperature threshold can resolve the issue. Further magnetically guided removal HNS from the brain or from the body tumor after therapy seems to be a relevant research topic to investigate in future.

\subsection{Ultrasound Triggered Hybrid Nanostructures}

Focused ultrasound sonication (FUS) combined with microbubbles (MB) can effectively increase the BBB permeability hence facilitating brain tumor therapy ${ }^{94}$. The effect 
is transient, non-invasive, reversible, and does not damage neural cells. When subjected to FUS, the tumour vasculature has enhanced permeability to chemotherapeutic drugs and there is a transient rupture of vascular barriers by FUS triggering. This is a result of the combined effect of oscillation in size and position of the MBs, the rupture of the MBs, microstreaming and radiation forces (Figure 7).

\subsubsection{Liposomes}

The earliest attempt at FUS-enhanced delivery for brain tumor involved 1,3-bis(2chloroethyl)-1- nitrosourea (BCNU) administered to rats with induced glioblastoma tumors ${ }^{95}$. FUS triggering significantly improved the BCNU penetration through the BBB in both the normal (by 340\%) and tumor implanted (by 202\%) brains without causing haemorrhaging. This novel physical triggering supports the viability of this treatment in clinical applications by increasing the chemotherapeutic payload delivered to the targeted brain tumor. Ligandconjugated hybrid nano liposomes triggered by FUS can facilitate an increased uptake of chemotherapeutic agents in brain tumors as well as improving the tumor-to contralateral brain ratio. Lipo-DOX in combination with ultrasound results in a unique pharmacokinetic profile. Yang et. al., evaluated the pharmacokinetics of targeted and untargeted Lipo-DOX hybrid structures in GBM-bearing mice ${ }^{96}$. The targeted particles were modified with human atherosclerotic plaque-specific peptide-1 (AP-1). When administered to the animals in combination with FUS, there was an enhancement in the uptake of the drug in the brain tumors.

\subsubsection{Organic-Inorganic HNS}

Dual-modality particles can simultaneously open the BBB and release the drug payload upon FUS exposure, and facilitate magnetic targeting to the brain tumor. To achieve this multimodality, novel HNS consisting of microbubbles with a superparamagnetic core loaded with doxorubicin (DOX-SPIO) have been developed ${ }^{97}$. These organic-inorganic HNS can serve as an effective drug carrier, burst under FUS exposure and release cargo into tumor as well as giving enhanced contrast for MR monitoring the distribution of the drug within the brain. The combination of FUS and magnetic targeting delivers therapeutic HNS across the blood-brain barrier to enter the brain tumor both passively and actively. Systemic administration of DOX in combination with using MRI-guided FUS with preformed microbubbles (Optison) has been shown to improve the DOX accumulation in glioma tissue ${ }^{98}$. Other groups have shown that modifications to the surface such as a polyethylene glycol (PEG) coating on particles loaded with cisplatin as an MRI -guided FUS modality in brain cancer 
enabled them to cross both the BBB and blood-tumor barriers, deliver payload under ultrasound triggering and also be detectable under MRI ${ }^{99}$. This novel HNS MRI -guided FUS theranostics may provide an effective new approach for cancer therapy, predominantly for preventing the recurrence of brain tumors.
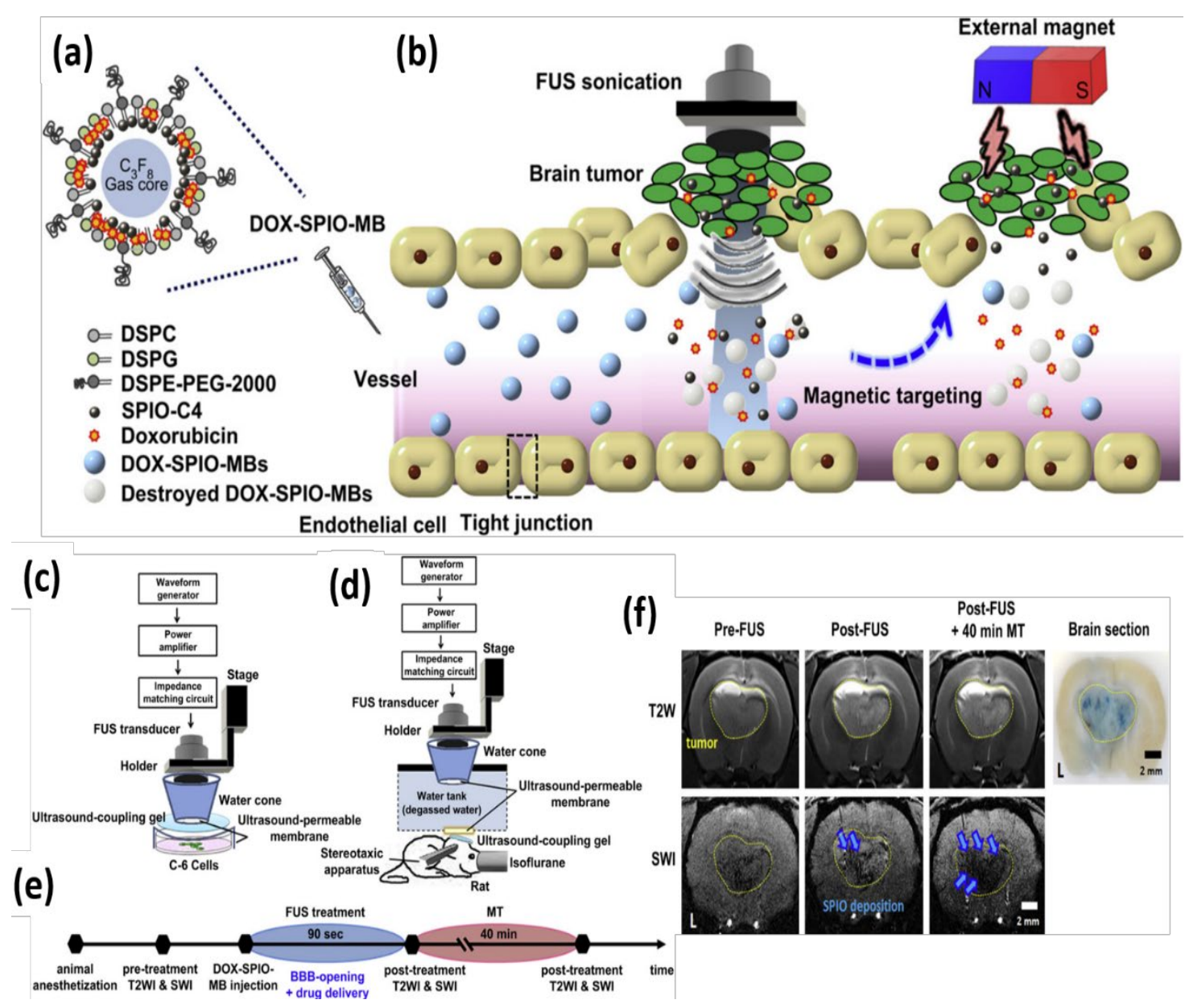

Figure 7 (A) Schematic illustration of DOX-SPIO-MBs. (B) Hypotheses for controlled release of DOX-SPIO-MBs into brain tissue triggered by FUS, and enhanced SPIO deposition using external MT. Blue circles: DOX-SPIO-MBs; White circles: Destroyed DOX-SPIO-MBs; Red/yellow octagons: DOX; gray circles: SPIO particles. (C) In vitro and (D) in vivo experimental setup. FUS was delivered to the tumor site in the left brain. (E) Time course of treatment and MRI monitoring. MRI T2W images (to confirm tumor location) and SWI images (to confirm SPIO deposition) obtained before and after FUS sonication with performing magnetic targeting for $40 \mathrm{~min}$. Arrow: Location of SPIO deposition. Tumor areas are delineated by yellow dotted lines. Reproduced with permission from ref 97. Copyright 2013 Elsevier. 
Poly(butyl cyanoacrylate)-based MBs, encapsulating ultrasmall SPIONs inside their shell, can be utilised to monitor BBB permeation by employing the FUS-MRI dual technique $^{100}$. External FUS triggering of HNS thus allows temporal and spatial control of the BBB opening, this is considered to be important for individualizing and improving drug delivery in case of brain tumors, Alzheimer's disease and Parkinson's disease. DOX was incorporated into liposomes making a perfect HNS for FUS triggered and MRI monitored brain cancer therapy, they found significant FUS-induced enhancements and consistent drug release from HNS in the brain tumors over the time ${ }^{101}$. Ultrasound-assisted delivery and targeting of poly(2-ethyl-butyl cyanoacrylate) polymeric HNS in normal brain parenchyma of a metastatic brain tumour model was achieved by using a novel FUS system, combining $1.1 \mathrm{MHz}$ magnetic resonance imaging (MRI) guided targeting of the treatment area ${ }^{102}$.

The FUS triggering of HNS is a targeted, noninvasive, and reversible procedure with superior precision to a selected area of interest in the deep brain using MR image guidance. A hybrid magnetic-graphene oxide carrying Epirubicin (EPI) for magnetic guided and FUS triggered has been designed that can deliver the drug to the brain tumor while being monitored by $\mathrm{MRI}^{103}$. These HNS can easily reach the local tumor lesion and deliver chemotherapeutic cargo, showing 14.7 times more accumulation in the targeted area compared to the surrounding healthy tissue. The particles may then be used as heat-generating materials to achieve targeted hyperthermia by triggering local tumor heating upon stimulation by extremely low-power FUS (LFUS), with a 20-50 fold decrease from high intensity FUS.

Very recently, the use of low-intensity ultrasound therapy termed as sonodynamic therapy (SDT) emerged as a novel approach for the treatment of brain and other cancerous tumors $^{104}$. SDT uses low-intensity ultrasound together with a sonosensitizer (molecule sensitive to ultrasound). The skin penetration depth of the high intensity ultrasound waves is limited and is inversely dependent to the frequency of the pressure wave, thereby its effect is limited to the stratum corneum (outermost layer of the skin) at high frequencies ${ }^{105}$. Glioma cell accumulated porphyrin derivatives, including 5-aminolevulinic acid, protoporphyrin IX and talaporfin sodium, are proposed as sonosensitizers in SDT by Houkin et.al., ${ }^{106}$. The proposed sonosensitizers enhanced the cytotoxicity of glioma cells under low intensity sonication, and then can be further improved by enhancing the degree of intracellular accumulation of sonosensitizer into glioma cells. Nevertheless, these sonosensitizers are strongly hydrophobic, and easily aggregate in a physiological environment. Thus limiting their effectiveness and negatively affecting their pharmacokinetic behaviour. In order to overcome such critical issues, the application of various type of HNS in combination with SDT shows a great potential ${ }^{107-}$ 
110. Newly introduced cell targeting hybrid nano-liposomes are implemented to improve the therapeutic outcome of SDT for brain cancer ${ }^{111}$. This novel HNS consists of iRGD modified DVDMS (also called sinoporphyrin sodium) and is conjugated into the targeting liposome (iRGD-Lipo-DVDMS). This formulation has boosted the sonodynamic effect through reactive oxygen species formation in response to focused ultrasound exposure as well as showing good in vivo biocompatibility and promising results in fluorescence image-guided sonodynamic brain cancer therapy. Researchers from Tokyo Women's Medical University conducted safety trials of experimental SDT combined with nanobots on a 12-year-old dog (natural canine patient) with a terminal-stage chondrosarcoma, this can be a revolutionary cancer therapy and the predicated the 5 year survival rate for Grade II glioma tumors was 93 $\%{ }^{112}$.

There are still a few obstacles in translating the HNS-based SDT from basic nanostructures to a clinical setting. For instance, the comprehensive in vivo cytotoxicity of many nanoformulations must still be clarified. Similarly, the biodistribution will need to be closely monitored and it is also be important to understand the pharmacokinetics of the HNS coupled with SDT when delivered to brain tumors. However, advances in the field are in progress as more in vitro and in vivo studies are being performed ${ }^{113,114}$ as well as SDT is further combined with photodynamic therapy ${ }^{107}$ to enhance the therapeutic outcome.

\subsubsection{Strengths and Challenges}

HNS designed to response FUS sonication in the presence of microbubbles are able to promote the effectiveness of chemotherapeutic cargo delivery in the brain and improving therapeutic potential. Compared to light and magnetic triggering FUS sonication creates transient rupture of vascular barriers by radiation forces generated by oscillations. This physical phenomenon allows HNS to overcome the BBB and subsequent increase in the tumor's vascular permeability. Tumor disruption by FUS is transient, reversible, and does not damage neural cells. FUS triggering of HNS thus, provide safer brain treatment over the light and magnetic remote activation and allows delivery of therapeutic or diagnostic agents to the brain tumor.

The FUS triggering of HNS is recently proposed brain theranostics approach, and hence there is wide scope for researchers to develop this newer phenomenon in depth before clinical translation. The neuronavigational guidance technique in neurosurgery or in neuro oncology provided new way of 3D images navigation of therapeutic cargoes. Designing an interface between FUS triggering of HNS and neuronavigator could allow better and more accurate brain 
tumor guidance and therapy. Neuronavigation-guided FUS triggering of HNS in the brain tumor has challenging but nanomedicine approach and has a huge potential as flexible medical tool for drug delivery across the BBB. However, more biomedical engineering development are required to predict the accuracy of neuronavigation in guiding FUS within clinical limit, without such targeted HNS FUS triggering in the tumor is impracticable.

\subsection{Electro-Magnetically Triggered Hybrid Nanostructures}

Electromagnetic triggering (EMT) has the potential to enable non-invasive controlled release of chemotherapeutic cargo and relies on using magneto-electric nanoparticles (MENPs). A brain tumor is surrounded by a complex neural network which is sensitive to external electric fields. EMT combined with MENPs can be utilised for brain stimulation or localized targeted brain tumor theranostics by using strong local electric charge oscillations induced by external fields that can directly interact with the neural network. This can allow for 'on demand' drug release in tumor by changing the electrostatic interaction or binding force between the nanoparticle surface and the attached drugs. These interactive forces are restrained by the magnetoelectric (ME) effect generated by an external magnetic field for remote control of the intrinsic local electric fields ${ }^{115}$.

\subsubsection{Organic-Inorganic HNS}

For the first time, K Yue et. al., and his group predicted the artificial stimulation of neurons using MENPs deep inside the brain by using computational programming ${ }^{116}$. This study demonstrated the concept of coupling neuronal electric signals to the magnetic dipoles of MENPs in a noninvasive technology that could be used to treat various brain disorders and brain tumors. Other 'on demand' systems used tumor growth inhibiting synthetic peptides bound onto the surface of MENPs ${ }^{117}$. The system comprised a central MENPs ( $\sim 30-\mathrm{nm}$ in size) of $\mathrm{CoFe}_{2} \mathrm{O}_{4} @ \mathrm{BaTiO}_{3}$, coated by growth hormone-releasing hormones antagonist of the MIA class (MIA690). The high-efficacy binding of MIA690 to MENPs is externally controlled. The peptide could be released on-demand by the application of direct current (d.c.) and alternating current (a.c.) magnetic fields,. Intracellular chemotherapeutic cargo release was found to be 11-times greater in cells treated with MIA690-loaded MENPs coupled with EMT compared with treatment with MIA690 alone $(\mathrm{p}<0.01)$. Drug-loaded liposomes and iron oxide nanosphere hybrid nanoparticles have also been developed for EMT in brain tumours ${ }^{118}$. Liposomal membranes on the iron oxide nanoparticle were shown to be responsive to electro- 
mechanical oscillations produced by external low power radiofrequency field (amplitude $\mathrm{B}=$ $2 \mathrm{mT}$, frequency $\mathrm{f}=10 \mathrm{kHz}, \mathrm{RF}$ power $=3-5$ Watts). These novel low power RF based EMT could remotely trigger site-specific rapid drug release from the hybrid nanochains ${ }^{119}$.

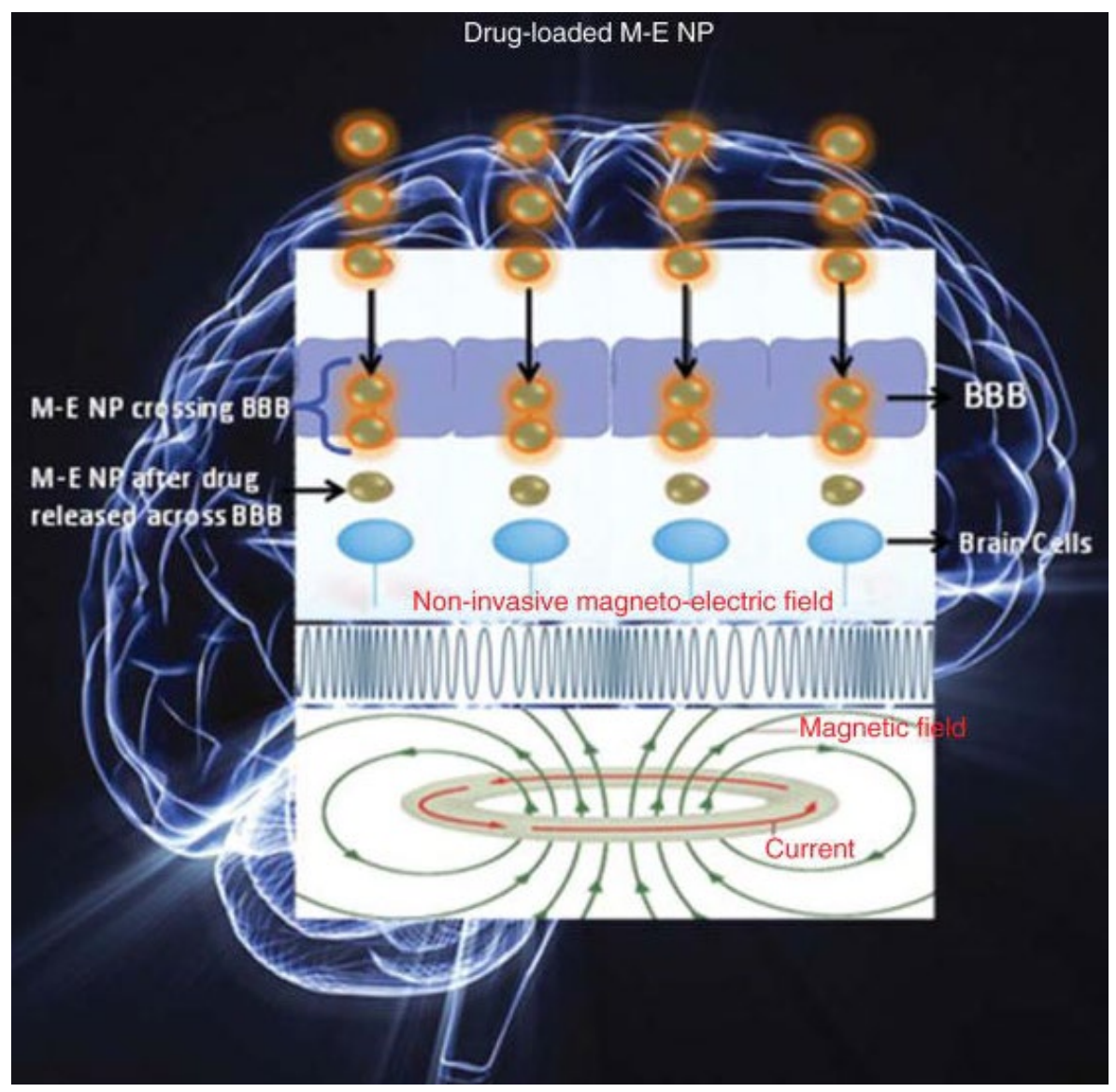

Figure 8. Proposed schematic of Magneto-electric nanocarriers based Anti-retroviral (ARV) drugs delivery across the BBB to treat the brain diseases and brain tumor efficiently. Reproduced with permission from ref 120. Copyright 2014 Informa UK, Ltd.

Most of the recent studies utilise MENPs of the same core-shell structure, i.e., $\mathrm{CoFe}_{2} \mathrm{O}_{4}-\mathrm{BaTiO}_{3}$. This MENPs based EMT approach could eventually be applied to other cancer types, due to the fundamental commonalities ${ }^{121}$. Future work could benefit from investigating other hybrid compositions, e.g., biodegradable organic materials loaded with inorganic NPs. In comparison to magnetic hyperthermia, the EMT based on MENPs allows on-demand release with no heat dissipation upon drug release (Figure 8). Additionally, 
development of MENPs and EMT techniques can result into dual targeting delivery system to a brain tumor and other brain diseases ${ }^{122}$, which is still in its early stage. Kaushik et.al., recently proposed novel hybrid nanocarriers in which MENPs which possess ideal properties can couple intrinsic electric and magnetic fields. Which could provide molecular composition selectivity, which would be a huge addition to the conventional diagnostic methods such as MRI and positron emission tomography-computed tomography for brain cancer or other brain diseases $^{120}$. MENPs based EMT can be further used for neuronal activation in the brain and MENPs could also provide MRI contrasting properties to gather molecular information of the adjacent brain tissue/microenvironment.

\subsubsection{Strengths and Challenges}

Hybrid MENPs are capable of on-demand drug release triggered by external magnetic or electric forces at target organ such as brain tumor. Thus, this novel HNS physical stimulation has significant scope in the neuro-oncology. EMT triggering allows magnetization and electrical polarization in a single phase of MENPs, which is advantageous for on demand cargo release. Designing novel MENPs with both piezoelectric properties and the ferroelectric phase change can advances the current therapeutic approach. The current challenges of formulation lie in the development of MENPs that improves electrical-magnetic responsiveness for ondemand drug release as well as biocompatibility and surface modifications for optimum drug loading/preservation/transmigration and tailoring of properties. In general, MENPs are core shell structures, wherein a magnetic core is preserved with a shell of desired electrical properties $^{123,124}$. The control on core size with thin shell and overall fine adjustment of the hybrid structure with phase fraction of MENPs formulation ensuing materials tunable properties, reproducible features are the most challenging requisite.

\section{Challenges, Conclusion and Future Outlooks}

\subsection{Challenges}

\subsubsection{HNS targeted delivery}

Despite significant progress in the understanding of the biological aspects of HNS and the development of novel delivery agents, clinicians are still struggling to manage targeted brain cancer therapeutics. The effective delivery of HNS along with therapeutic agents to brain tumor tissues is hindered due to the presence of two main obstacles, the first is the BBB and other one is blood-cerebrospinal fluid barrier ${ }^{125}$. Apart from this, highly-expressed active efflux pumps 
or transporters at the luminal membrane of the brain endothelium can dynamically expel foreign substances such as HNS back to the blood stream ${ }^{126}$. The BBB has been found to be an interface which dynamically controls entry to the brain from the blood preventing $95 \%$ of molecules from being suitable for passive targeting ${ }^{127}$. These advances emphasize the need for reconsidering some concepts of nanomedicine based brain drug delivery, and also reveal great opportunities for new strategies to deliver drugs into the brain. Due to the heterogeneity in the brain physiological conditions, the externally controlled stimuli-responsive targeting strategies might be more favourable, allowing for targeting different pathological micro-environments. Among the all physical modalities discussed in above sections, magnetic field targeting of HNS is shown to be an advantageous approach when compared with the molecular targeted approach that could allow guidance of MNPs across the $\mathrm{BBB}^{128}$. The magnetic force applied by external magnet placed externally near the surface of the scull can be used to drag MNPs though the BBB. Stepwise targeting of bio-functionalized MNPs to brain tumor followed by magnetically guided transport through the $\mathrm{BBB}$ using an external magnetic force may prove a novel mechanism for targeted therapy of macromolecules to the brain. The basic principles of magnetic targeting and detailed discussion on the potentials of magnetic field-mediated drug delivery can be found in literature ${ }^{129-131}$.

Another major challenge in targeted HNS delivery is to understand the dynamic change in brain tumor. When tumor cells reach a certain level, the BBB is damaged and this can lead to the formation of the blood-brain tumor barrier (BBTB). The BBTB is formed by new blood vessels (brain tumor capillary) and is distinct from the BBB. Thus, the combination of the BBB and the BBTB form a major barrier for targeted therapeutic cargo delivery to brain tumor. To overcome these issues, ultrasound facilitated drug delivery became an attractive technique to facilitate HNS transport into brain tumor across the BBB and BBTB. The combination of FUS and microbubbles enhances the permeability of the BBTB in brain tumors as well as disrupting the $\mathrm{BBB}^{127}$. Hybrid PLGA nanovehicles holding doxorubicin/perfluorooctyl bromide (ANP$\mathrm{D} / \mathrm{P}$ ) was designed to target the glioblastoma sites and increase the intratumoral drug accumulation, further FUS trigger on-demand drug release in the tumor, and enhance glioblastoma therapy at clinical stage ${ }^{132}$. Similarly, tumor-homing echogenic glycol chitosanHNS presented FUS-triggered tumor uptake and drug release profile through the external US irradiation $^{133}$. Thus, novel HNS coupled with ultrasound can overcome the BBTB and the BBB and could achieve site- and time-specific chemotherapeutic cargo release for efficient brain cancer treatment. 


\subsubsection{HNS surface properties}

Surface physical properties of the HNS are a fundamental characteristic that determines the passive targeting and biodistribution within brain tumors. HNS within the size range of 10-100 $\mathrm{nm}$ is the most favourable for accessing hyper-vascularized, leaky, and compromised lymphatic drainage system in a brain tumor as well as passively target and access the intratumoral space while being denied access to healthy brain tissue ${ }^{134}$. In other words, optimum sized (10-100 $\mathrm{nm}$ ) HNS accumulate selectively in the brain tumor due to enhanced permeability and retention (EPR) effect. This EPR effect is not observed for small molecular weight chemotherapeutic molecules and they rely on free diffusion and thus internalised by normal and diseased tissue. However, NPs entry in physiological environment of the body, proteins from the bloodstream get absorbed on the NP surface and forms 'protein corona' This protein corona restricts NPs localized entry to tumor site and accelerates blood clearance mostly by liver and spleen. This challenge has been recently overcome by developing novel HNS consisting anti-fouling agents such as poly(ethylene-glycol) (PEG) and D-a-tocopherol polyethylene glycol 1000 succinate (TPGS, Tocofersolan) ${ }^{135}$. The surface charge of HNS is another crucial factor for penetration into a tumor. Mediating the surface charge of -15 to $-45 \mathrm{mV}$ can greatly improve the brain accumulation of HNS. By altering the surface charge and hydrophobicity one can influence the half-life and localization of HNS during the circulation. Dense PEG coating or PEGylation process alters the surface charge of HNS which can prolong the blood circulation half-life, subsequently increasing the chance of reaching distant brain tumor cells ${ }^{136}$.

\subsubsection{Other Challenges}

Whilst the basic understanding of the function of HNS is understood, there are still hands-on facts that need to be addressed. The most significant fact is the response time by which a remote stimulus conveys a triggering in the material, e.g. in stimuli-sensitive hydrogels these changes occur on a reasonably slow time-scale ${ }^{137}$ and therefore fast-acting hybrid systems are required. On the other hand polymer conjugated magnetic HNS can overcome this short-comings. Ideally, a remotely triggered HNS must have fast response time to physical stimulus and must be biocompatible, not invoke a host inflammatory response and have an optimum size $(\sim 10-20 \mathrm{~nm})$ to cross the BBB. With the increasing requirements on preclinical to clinical translation, the main demand is to delivering right amount of tumor therapeutic cargo at the right time and right place within brain tumor lesions. Remotely triggered HNS can accomplishes these good promise of benefit to the brain tumor patients, but presents huge challenges. These preliminary challenges are: (i) zero premature release in blood 
stream, (ii) pulsatile or random uncontrolled release in tumor microenvironment, (iii) targeting delivery and minimal heating up to the surroundings in photothermal, photodynamic, magneto - thermal therapies, (iv) providing quantitative, real-time, and three-dimensional (3D) continuous therapy guidance in brain cancer as well as chemical and molecular heterogeneity in all cancer cells that have invaded normal brain with 85 billion neurons. Surface Enhanced Raman Spectroscopy (SERS) active HNS has a strong potential of normal and cancerous brain imaging for clinical translation. Recently, such novel technology based on SERS nanoparticles and a hand-held Raman scanner has been successfully tested in preclinical stage further that can guide brain tumor resection in the operating room. ${ }^{138}$

\subsection{Conclusion}

A nanovehicle drug delivery system responding to cellular local stimuli, such as $\mathrm{pH}$, temperature, reductive agents activation i.e. enzymes, could effectively provide passive mode desirable release but fail in disease treatment following the biological rhythms of brain tumor. Compared to other organs or tumors, brain tumors are surrounded by highly vascular tissues which provide a high surface area of $20 \mathrm{~m}^{2}$ and a total length of $\sim 400$ miles $^{139}$. This complex physiology of the brain tumor over other type of tumors hinders the effective therapeutics outcome with conventional drugs and therapies. Undoubtedly, HNS aimed for remotely triggered release could offer an alternative solution as on demand and efficient brain cancer theranostics, because HNS are widely more functional than other types of nanostructures thus, more promising as a possible nanotheranostic for brain cancer. These enhanced functionalities of HNS include: (i) HNS have distinct surface properties that can further facilitate the encapsulation of large therapeutic molecules, for example, siRNA, cDNA and polypeptides, (ii) HNS enhances delivery of therapeutic cargos to the brain tumor by the transport of MNPs, polymers or liposomes across the BBB aided by an extra cranially applied magnetic force, ultrasound or other external triggers, (iii) Remotely triggered HNS facilitate stepwise targeting and delivery of macromolecules to the brain tumor through brain capillary endothelial cells followed by magnetic/ultrasound/light guided transport through the BBB and may prove a novel mechanism for remotely controlling targeted brain cancer therapy. Moreover, remote triggering of these HNS more advantageous in terms of improved therapeutics efficiency, spatial and temporal controllability over the chemically stimulated release, the lack of undesirable adverse effects and minimal cargo detachment during the in vivo delivery, etc. The compilation of work described here establishes that multicomponent "smart" hybrid 
nanosystems that integrate chemotherapeutic agents are capable of triggering cargo release at brain tumor sites when activated by external stimuli. These novel multimodality can be extremely potent in understanding cancer through real-time observation of drug delivery, release, and activity for non-conventional cancer therapies.

\subsection{Future Outlooks}

Future research perspectives should be directed to resolve following issues for successful preclinical to clinical translation of remotely triggered HNS modalities such as:

(i) Resolving difficulty in visualizing brain tumor margins intraoperatively remains a major issue in the achievement of gross total tumor resection (diagnosis) and, thus, better clinical outcome of high-grade tumor patients.

(ii) Coupled to this is a lack of ideal anticancer drugs (therapeutics) that can be tailored on the basis of the patient's genetic make-up (mainly gender and ethnicity; also termed pharmacogenetics ${ }^{140}$ ) as well as individual tumor conditions.

(iii) Post-treatment or post-surgery of tumor imaging (therapy monitoring) by MRI/CT or optical techniques often lacks inadequate sensitivity, specificity and spatial resolution to define the actual tumor borders during surgery or after (radio) therapy because of brain shift.

These are just some of the future perspectives that need to be addressed if remotely triggered theranostic treatments are to be used for neuro oncology. Next-generation HNS, proteins, biomolecules and probes for neural interfacing around the brain tumor are also necessary. ${ }^{141}$ The overall HNS iterative design from skilled researchers can scale up robust and reproducible nano architecture in remotely triggered brain cancer theranostics. Understanding the remotely triggered approaches, their modality, and the role of cross-disciplinary development will support new neuro oncological therapies and prostheses and provide material scientist, neuroscientists, oncologist, radiologists and neurologists with unprecedented access to the brain tumor.

\section{References:}

(1) Global Cancer Observatory Report, https://gco.iarc.fr/databases.php http://ci5.iarc.fr/CI5plus/Pages/download.aspx (accessed Sep 23, 2018).

(2) de Robles, P.; Fiest, K. M.; Frolkis, A. D.; Pringsheim, T.; Atta, C.; St GermaineSmith, C.; Day, L.; Lam, D.; Jette, N. The Worldwide Incidence and Prevalence of Primary Brain Tumors: A Systematic Review and Meta-Analysis. Neuro. Oncol. 2015, 17 (6), 776-783.

(3) Arora, R. S.; Alston, R. D.; Eden, T. O. B.; Estlin, E. J.; Moran, A.; Birch, J. M. AgeIncidence Patterns of Primary CNS Tumors in Children, Adolescents, and Adults in 
England. Neuro. Oncol. 2009, 11 (4), 403-413.

(4) Mangraviti, A.; Gullotti, D.; Tyler, B.; Brem, H. Nanobiotechnology-Based Delivery Strategies: New Frontiers in Brain Tumor Targeted Therapies. J. Control. Release 2016, 240, 443-453.

(5) Zhu, J.-J.; Wong, E. T. Personalized Medicine for Glioblastoma: Current Challenges and Future Opportunities. Curr. Mol. Med. 2013, 13 (3), 358-367.

(6) Tricoli, J. V.; Seibel, N. L.; Blair, D. G.; Albritton, K.; Hayes-Lattin, B. Unique Characteristics of Adolescent and Young Adult Acute Lymphoblastic Leukemia, Breast Cancer, and Colon Cancer. JNCI J. Natl. Cancer Inst. 2011, 103 (8), 628-635.

(7) Sonali; Viswanadh, M. K.; Singh, R. P.; Agrawal, P.; Mehata, A. K.; Pawde, D. M.; Narendra; Sonkar, R.; Muthu, M. S. Nanotheranostics: Emerging Strategies for Early Diagnosis and Therapy of Brain Cancer. Nanotheranostics 2018, 2 (1), 70-86.

(8) Alentorn, A.; Sanson, M.; Idbaih, A. Oligodendrogliomas. Curr. Opin. Oncol. 2012, 24 (6), 687-693.

(9) Bhojani, M. S.; Van Dort, M.; Rehemtulla, A.; Ross, B. D. Targeted Imaging and Therapy of Brain Cancer Using Theranostic Nanoparticles. Mol. Pharm. 2010, 7 (6), 1921-1929.

(10) Zhou, Y.; Peng, Z.; Seven, E. S.; Leblanc, R. M. Crossing the Blood-Brain Barrier with Nanoparticles. J. Control. Release 2018, 270, 290-303.

(11) Zhang, T.-T.; Li, W.; Meng, G.; Wang, P.; Liao, W. Strategies for Transporting Nanoparticles across the Blood-brain Barrier. Biomater. Sci. 2016, 4 (2), 219-229.

(12) He, Q.; Liu, J.; Liang, J.; Liu, X.; Li, W.; Liu, Z.; Ding, Z.; Tuo, D. Towards Improvements for Penetrating the Blood-Brain Barrier-Recent Progress from a Material and Pharmaceutical Perspective. Cells 2018, 7 (4), 24.

(13) Liu, B.; Li, C.; Cheng, Z.; Hou, Z.; Huang, S.; Lin, J. Functional Nanomaterials for Near-Infrared-Triggered Cancer Therapy. Biomater. Sci. 2016, 4 (6), 890-909.

(14) Yang, Y.; Aw, J.; Xing, B. Nanostructures for NIR Light-Controlled Therapies. Nanoscale 2017, 9 (11), 3698-3718.

(15) Chakroun, R. W.; Zhang, P.; Lin, R.; Schiapparelli, P.; Quinones-Hinojosa, A.; Cui, H. Nanotherapeutic Systems for Local Treatment of Brain Tumors. Wiley Interdiscip. Rev. Nanomedicine Nanobiotechnology 2018, 10 (1), e1479.

(16) Thomas, A. A.; Omuro, A. Current Role of Anti-Angiogenic Strategies for Glioblastoma. Curr. Treat. Options Oncol. 2014, 15 (4), 551-566.

(17) Lieberman, N. A. P.; Vitanza, N. A.; Crane, C. A. Immunotherapy for Brain Tumors: Understanding Early Successes and Limitations. Expert Rev. Neurother. 2018, 18 (3), 251-259.

(18) Quirk, B. J.; Brandal, G.; Donlon, S.; Vera, J. C.; Mang, T. S.; Foy, A. B.; Lew, S. M.; Girotti, A. W.; Jogal, S.; LaViolette, P. S.; Connelly, J. M.; Whelan, H. T. Photodynamic Therapy (PDT) for Malignant Brain Tumors - Where Do We Stand? Photodiagnosis Photodyn. Ther. 2015, 12 (3), 530-544.

(19) Sun, J.; Guo, M.; Pang, H.; Qi, J.; Zhang, J.; Ge, Y. Treatment of Malignant Glioma Using Hyperthermia. Neural Regen. Res. 2013, 8 (29), 2775-2782.

(20) Murphy, A. M.; Rabkin, S. D. Current Status of Gene Therapy for Brain Tumors. Transl. Res. 2013, 161 (4), 339-354.

(21) Sneider, A.; VanDyke, D.; Paliwal, S.; Rai, P. Remotely Triggered Nano-Theranostics For Cancer Applications. Nanotheranostics 2017, 1 (1), 1-22.

(22) Bohara, R. A.; Thorat, N. D.; Pawar, S. H. Role of Functionalization: Strategies to Explore Potential Nano-Bio Applications of Magnetic Nanoparticles. RSC Adv. 2016, $6(50)$.

(23) Thorat, N. D.; Bohara, R. A.; Noor, M. R.; Dhamecha, D.; Soulimane, T.; Tofail, S. A. 
M. Effective Cancer Theranostics with Polymer Encapsulated Superparamagnetic Nanoparticles: Combined Effects of Magnetic Hyperthermia and Controlled Drug Release. ACS Biomater. Sci. Eng. 2017, 3 (7).

(24) Sailor, M. J.; Park, J.-H. Hybrid Nanoparticles for Detection and Treatment of Cancer. Adv. Mater. 2012, 24 (28), 3779-3802.

(25) Li, M.; Luo, Z.; Zhao, Y. Self-Assembled Hybrid Nanostructures: Versatile Multifunctional Nanoplatforms for Cancer Diagnosis and Therapy. Chem. Mater. 2018, 30 (1), 25-53.

(26) Raizer, J. Issues in Developing Drugs for Primary Brain Tumors: Barriers and Toxicities. Toxicol. Pathol. 2011, 39 (1), 152-157.

(27) Fisusi, F. A.; Schätzlein, A. G.; Uchegbu, I. F. Nanomedicines in the Treatment of Brain Tumors. Nanomedicine 2018, 13 (6), 579-583.

(28) Thorat, N. D.; Bohara, R. A.; Tofail, S. A. M.; Alothman, Z. A.; Shiddiky, M. J. A.; A Hossain, M. S.; Yamauchi, Y.; Wu, K. C.-W. Superparamagnetic Gadolinium Ferrite Nanoparticles with Controllable Curie Temperature - Cancer Theranostics for MRImaging-Guided Magneto-Chemotherapy. Eur. J. Inorg. Chem. 2016, 2016 (28), 4586-4597.

(29) Chan, A.; Orme, R. P.; Fricker, R. A.; Roach, P. Remote and Local Control of Stimuli Responsive Materials for Therapeutic Applications. Adv. Drug Deliv. Rev. 2013, 65 (4), 497-514.

(30) Liu, J.; Detrembleur, C.; Mornet, S.; Jérôme, C.; Duguet, E. Design of Hybrid Nanovehicles for Remotely Triggered Drug Release: An Overview. J. Mater. Chem. B 2015, 3 (30), 6117-6147.

(31) Nair, R.; Maseeh, A. Vitamin D: The \&quot;Sunshine\&quot; Vitamin. J. Pharmacol. Pharmacother. 2012, 3 (2), 118-126.

(32) Zhang, P.; Hu, C.; Ran, W.; Meng, J.; Yin, Q.; Li, Y. Recent Progress in LightTriggered Nanotheranostics for Cancer Treatment. Theranostics 2016, 6 (7), 948-968.

(33) Kim, H.; Chung, K.; Lee, S.; Kim, D. H.; Lee, H. Near-Infrared Light-Responsive Nanomaterials for Cancer Theranostics. Wiley Interdiscip. Rev. Nanomedicine Nanobiotechnology 2016, 8 (1), 23-45.

(34) Quirk, B. J.; Brandal, G.; Donlon, S.; Vera, J. C.; Mang, T. S.; Foy, A. B.; Lew, S. M.; Girotti, A. W.; Jogal, S.; LaViolette, P. S.; Connelly, J. M.; Whelan, H. T.

Photodynamic Therapy (PDT) for Malignant Brain Tumors - Where Do We Stand? Photodiagnosis Photodyn. Ther. 2015, 12 (3), 530-544.

(35) Li, Z.; Ye, E.; David; Lakshminarayanan, R.; Loh, X. J. Recent Advances of Using Hybrid Nanocarriers in Remotely Controlled Therapeutic Delivery. Small 2016, 12 (35), 4782-4806.

(36) Seekell, K.; Lewis, S.; Wilson, C.; Li, S.; Grant, G.; Wax, A. Feasibility Study of Brain Tumor Delineation Using Immunolabeled Gold Nanorods. Biomed. Opt. Express 2013, 4 (11), 2284-2295.

(37) Kim, J. E.; Choi, J. H.; Colas, M.; Kim, D. H.; Lee, H. Gold-Based Hybrid Nanomaterials for Biosensing and Molecular Diagnostic Applications. Biosens. Bioelectron. 2016, 80, 543-559.

(38) Hirschberg, H.; Madsen, S. J. Cell Mediated Photothermal Therapy of Brain Tumors. J. Neuroimmune Pharmacol. 2017, 12 (1), 99-106.

(39) Gao, X.; Yue, Q.; Liu, Z.; Ke, M.; Zhou, X.; Li, S.; Zhang, J.; Zhang, R.; Chen, L.; Mao, Y.; Li, C. Guiding Brain-Tumor Surgery via Blood-Brain-Barrier-Permeable Gold Nanoprobes with Acid-Triggered MRI/SERRS Signals. Adv. Mater. 2017, 29 (21), 1603917.

(40) Meyers, J. D.; Cheng, Y.; Broome, A.-M.; Agnes, R. S.; Schluchter, M. D.; 
Margevicius, S.; Wang, X.; Kenney, M. E.; Burda, C.; Basilion, J. P. Peptide-Targeted Gold Nanoparticles for Photodynamic Therapy of Brain Cancer. Part. Part. Syst. Charact. Meas. Descr. Part. Prop. Behav. powders other disperse Syst. 2015, 32 (4), 448-457.

(41) Dixit, S.; Novak, T.; Miller, K.; Zhu, Y.; Kenney, M. E.; Broome, A.-M. Transferrin Receptor-Targeted Theranostic Gold Nanoparticles for Photosensitizer Delivery in Brain Tumors. Nanoscale 2015, 7 (5), 1782-1790.

(42) Lee, C.; Hwang, H. S.; Lee, S.; Kim, B.; Kim, J. O.; Oh, K. T.; Lee, E. S.; Choi, H.-G.; Youn, Y. S. Rabies Virus-Inspired Silica-Coated Gold Nanorods as a Photothermal Therapeutic Platform for Treating Brain Tumors. Adv. Mater. 2017, 29 (13), 1605563.

(43) Yuan, H.; Wilson, C. M.; Xia, J.; Doyle, S. L.; Li, S.; Fales, A. M.; Liu, Y.; Ozaki, E.; Mulfaul, K.; Hanna, G.; Palmer, G. M.; Wang, L. V; Grant, G. A.; Vo-Dinh, T.

Plasmonics-Enhanced and Optically Modulated Delivery of Gold Nanostars into Brain Tumor. Nanoscale 2014, 6 (8), 4078-4082.

(44) Tomitaka, A.; Arami, H.; Huang, Z.; Raymond, A.; Rodriguez, E.; Cai, Y.; Febo, M.; Takemura, Y.; Nair, M. Hybrid Magneto-Plasmonic Liposomes for Multimodal Image-Guided and Brain-Targeted HIV Treatment. Nanoscale 2017, 10 (1), 184-194.

(45) Day, E. S.; Thompson, P. A.; Zhang, L.; Lewinski, N. A.; Ahmed, N.; Drezek, R. A.; Blaney, S. M.; West, J. L. Nanoshell-Mediated Photothermal Therapy Improves Survival in a Murine Glioma Model. J. Neurooncol. 2011, 104 (1), 55-63.

(46) Wang, J.; Zhao, H.; Zhou, Z.; Zhou, P.; Yan, Y.; Wang, M.; Yang, H.; Zhang, Y.; Yang, S. MR/SPECT Imaging Guided Photothermal Therapy of Tumor-Targeting $\mathrm{Fe} @ \mathrm{Fe}_{3} \mathrm{O}_{4}$ Nanoparticles in Vivo with Low Mononuclear Phagocyte Uptake. ACS Appl. Mater. Interfaces 2016, 8 (31), 19872-19882.

(47) Chen, M.-H.; Jenh, Y.-J.; Wu, S.-K.; Chen, Y.-S.; Hanagata, N.; Lin, F.-H. NonInvasive Photodynamic Therapy in Brain Cancer by Use of Tb3+-Doped LaF3 Nanoparticles in Combination with Photosensitizer Through X-Ray Irradiation: A Proof-of-Concept Study. Nanoscale Res. Lett. 2017, 12 (1), 62.

(48) Zhang, H.; Zhang, W.; Zhou, Y.; Jiang, Y.; Li, S. Dual Functional Mesoporous Silicon Nanoparticles Enhance the Radiosensitivity of VPA in Glioblastoma. Transl. Oncol. 2017, 10 (2), 229-240.

(49) Benachour, H.; Sève, A.; Bastogne, T.; Frochot, C.; Vanderesse, R.; Jasniewski, J.; Miladi, I.; Billotey, C.; Tillement, O.; Lux, F.; Barberi-Heyob, M. Multifunctional Peptide-Conjugated Hybrid Silica Nanoparticles for Photodynamic Therapy and MRI. Theranostics 2012, 2 (9), 889-904.

(50) Tang, W.; Xu, H.; Kopelman, R.; A. Philbert, M. Photodynamic Characterization and In Vitro Application of Methylene Blue-Containing Nanoparticle Platforms $\uparrow$.

Photochem. Photobiol. 2005, 81 (2), 242.

(51) Iijima, S. Helical Microtubules of Graphitic Carbon. Nature 1991, 354 (6348), 56-58.

(52) Guo, Q.; Shen, X.; Li, Y.; Xu, S. Carbon Nanotubes-Based Drug Delivery to Cancer and Brain. Curr. Med. Sci. 2017, 37 (5), 635-641.

(53) Zhao, D.; Alizadeh, D.; Zhang, L.; Liu, W.; Farrukh, O.; Manuel, E.; Diamond, D. J.; Badie, B. Carbon Nanotubes Enhance CpG Uptake and Potentiate Antiglioma Immunity. Clin. Cancer Res. 2011, 17 (4), 771-782.

(54) Eldridge, B. N.; Bernish, B. W.; Fahrenholtz, C. D.; Singh, R. Photothermal Therapy of Glioblastoma Multiforme Using Multiwalled Carbon Nanotubes Optimized for Diffusion in Extracellular Space. ACS Biomater. Sci. Eng. 2016, 2 (6), 963-976.

(55) Hong, E. J.; Choi, D. G.; Shim, M. S. Targeted and Effective Photodynamic Therapy for Cancer Using Functionalized Nanomaterials. Acta Pharm. Sin. B 2016, 6 (4), 297 307. 
(56) Su, S.; Wang, J.; Vargas, E.; Wei, J.; Martínez-Zaguilán, R.; Sennoune, S. R.; Pantoya, M. L.; Wang, S.; Chaudhuri, J.; Qiu, J. Porphyrin Immobilized Nanographene Oxide for Enhanced and Targeted Photothermal Therapy of Brain Cancer. ACS Biomater. Sci. Eng. 2016, 2 (8), 1357-1366.

(57) Cardano, F.; Frasconi, M.; Giordani, S. Photo-Responsive Graphene and Carbon Nanotubes to Control and Tackle Biological Systems. Front. Chem. 2018, 6, 102.

(58) Hong, G.; Diao, S.; Chang, J.; Antaris, A. L.; Chen, C.; Zhang, B.; Zhao, S.; Atochin, D. N.; Huang, P. L.; Andreasson, K. I.; Kuo, C. J.; Dai, H. Through-Skull Fluorescence Imaging of the Brain in a New near-Infrared Window. Nat. Photonics 2014, 8 (9), 723-730.

(59) Tsai, Y.-C.; Vijayaraghavan, P.; Chiang, W.-H.; Chen, H.-H.; Liu, T.-I.; Shen, M.-Y.; Omoto, A.; Kamimura, M.; Soga, K.; Chiu, H.-C. Targeted Delivery of Functionalized Upconversion Nanoparticles for Externally Triggered Photothermal/Photodynamic Therapies of Brain Glioblastoma. Theranostics 2018, 8 (5), 1435-1448.

(60) Ni, D.; Zhang, J.; Bu, W.; Xing, H.; Han, F.; Xiao, Q.; Yao, Z.; Chen, F.; He, Q.; Liu, J.; Zhang, S.; Fan, W.; Zhou, L.; Peng, W.; Shi, J. Dual-Targeting Upconversion Nanoprobes across the Blood-Brain Barrier for Magnetic Resonance/Fluorescence Imaging of Intracranial Glioblastoma. ACS Nano 2014, 8 (2), 1231-1242.

(61) Jiang, Y.; Upputuri, P. K.; Xie, C.; Lyu, Y.; Zhang, L.; Xiong, Q.; Pramanik, M.; Pu, K. Broadband Absorbing Semiconducting Polymer Nanoparticles for Photoacoustic Imaging in Second Near-Infrared Window. Nano Lett. 2017, 17 (8), 4964-4969.

(62) Hao, Y.; Wang, L.; Zhao, Y.; Meng, D.; Li, D.; Li, H.; Zhang, B.; Shi, J.; Zhang, H.; Zhang, Z.; Zhang, Y. Targeted Imaging and Chemo-Phototherapy of Brain Cancer by a Multifunctional Drug Delivery System. Macromol. Biosci. 2015, 15 (11), 1571-1585.

(63) Jiang, F.; Lilge, L.; Grenier, J.; Li, Y.; Wilson, M. D.; Chopp, M. Photodynamic Therapy of U87 Human Glioma in Nude Rat Using Liposome-Delivered Photofrin. Lasers Surg. Med. 1998, 22 (2), 74-80.

(64) Sadasivam, M.; Avci, P.; Gupta, G. K.; Lakshmanan, S.; Chandran, R.; Huang, Y.-Y.; Kumar, R.; Hamblin, M. R. Self-Assembled Liposomal Nanoparticles in Photodynamic Therapy. Eur. J. Nanomedicine 2013, 5 (3), 115-129.

(65) Chen, Q.; Liang, C.; Sun, X.; Chen, J.; Yang, Z.; Zhao, H.; Feng, L.; Liu, Z. H2O2Responsive Liposomal Nanoprobe for Photoacoustic Inflammation Imaging and Tumor Theranostics via in Vivo Chromogenic Assay. Proc. Natl. Acad. Sci. U. S. A. 2017, 114 (21), 5343-5348.

(66) Carling, C.-J.; Boyer, J.-C.; Branda, N. R. Remote-Control Photoswitching Using NIR Light. J. Am. Chem. Soc. 2009, 131 (31), 10838-10839.

(67) Shen, Y.; Shuhendler, A. J.; Ye, D.; Xu, J.-J.; Chen, H.-Y. Two-Photon Excitation Nanoparticles for Photodynamic Therapy. Chem. Soc. Rev. 2016, 45 (24), 6725-6741.

(68) Gao, D.; Agayan, R. R.; Xu, H.; Philbert, M. A.; Kopelman, R. Nanoparticles for TwoPhoton Photodynamic Therapy in Living Cells. Nano Lett. 2006, 6 (11), 2383-2386.

(69) Qian, J.; Wang, D.; Cai, F.; Zhan, Q.; Wang, Y.; He, S. Photosensitizer Encapsulated Organically Modified Silica Nanoparticles for Direct Two-Photon Photodynamic Therapy and In Vivo Functional Imaging. Biomaterials 2012, 33 (19), 4851-4860.

(70) Alifu, N.; Yan, L.; Zhang, H.; Zebibula, A.; Zhu, Z.; Xi, W.; Roe, A. W.; Xu, B.; Tian, W.; Qian, J. Organic Dye Doped Nanoparticles with NIR Emission and Biocompatibility for Ultra-Deep in Vivo Two-Photon Microscopy under 1040 Nm Femtosecond Excitation. Dye. Pigment. 2017, 143, 76-85.

(71) Liu, H.-W.; Liu, Y.; Wang, P.; Zhang, X.-B. Molecular Engineering of Two-Photon Fluorescent Probes for Bioimaging Applications. Methods Appl. Fluoresc. 2017, 5 (1), 012003 . 
(72) Mahmoudi, K.; Hadjipanayis, C. G. The Application of Magnetic Nanoparticles for the Treatment of Brain Tumors. Front. Chem. 2014, 2, 109.

(73) Hayashi, K.; Nakamura, M.; Miki, H.; Ozaki, S.; Abe, M.; Matsumoto, T.; Sakamoto, W.; Yogo, T.; Ishimura, K. Magnetically Responsive Smart Nanoparticles for Cancer Treatment with a Combination of Magnetic Hyperthermia and Remote-Control Drug Release. Theranostics 2014, 4 (8), 834-844.

(74) Tietze, R.; Lyer, S.; Dürr, S.; Alexiou, C. Nanoparticles for Cancer Therapy Using Magnetic Forces. Nanomedicine 2012, 7 (3), 447-457.

(75) Chertok, B.; David, A. E.; Huang, Y.; Yang, V. C. Glioma Selectivity of Magnetically Targeted Nanoparticles: A Role of Abnormal Tumor Hydrodynamics. J. Control. Release 2007, 122 (3), 315-323.

(76) Fontes de Paula Aguiar, M.; Bustamante Mamani, J.; Klei Felix, T.; Ferreira dos Reis, R.; Rodrigues da Silva, H.; Nucci, L. P.; Nucci-da-Silva, M. P.; Gamarra, L. F. Magnetic Targeting with Superparamagnetic Iron Oxide Nanoparticles for in Vivo Glioma. Nanotechnol. Rev. 2017, 6 (5), 449-472.

(77) Shah, B. P.; Pasquale, N.; De, G.; Tan, T.; Ma, J.; Lee, K.-B. Core-Shell NanoparticleBased Peptide Therapeutics and Combined Hyperthermia for Enhanced Cancer Cell Apoptosis. ACS Nano 2014, 8 (9), 9379-9387.

(78) Chertok, B.; David, A. E.; Yang, V. C. Brain Tumor Targeting of Magnetic Nanoparticles for Potential Drug Delivery: Effect of Administration Route and Magnetic Field Topography. J. Control. Release 2011, 155 (3), 393-399.

(79) Chertok, B.; Moffat, B. A.; David, A. E.; Yu, F.; Bergemann, C.; Ross, B. D.; Yang, V. C. Iron Oxide Nanoparticles as a Drug Delivery Vehicle for MRI Monitored Magnetic Targeting of Brain Tumors. Biomaterials 2008, 29 (4), 487-496.

(80) Fang, J.-H.; Lai, Y.-H.; Chiu, T.-L.; Chen, Y.-Y.; Hu, S.-H.; Chen, S.-Y. Magnetic Core-Shell Nanocapsules with Dual-Targeting Capabilities and Co-Delivery of Multiple Drugs to Treat Brain Gliomas. Adv. Healthc. Mater. 2014, 3 (8), 1250-1260.

(81) Lal, S.; Verma, J.; Van Noorden, C. J. F. Nanoparticles for Hyperthermic Therapy: Synthesis Strategies and Applications in Glioblastoma. Int. J. Nanomedicine 2014, 9, 2863.

(82) Hemery, G.; Genevois, C.; Couillaud, F.; Lacomme, S.; Gontier, E.; Ibarboure, E.; Lecommandoux, S.; Garanger, E.; Sandre, O. Monocore vs. Multicore Magnetic Iron Oxide Nanoparticles: Uptake by Glioblastoma Cells and Efficiency for Magnetic Hyperthermia. Mol. Syst. Des. Eng. 2017, 2 (5), 629-639.

(83) Maier-Hauff, K.; Ulrich, F.; Nestler, D.; Niehoff, H.; Wust, P.; Thiesen, B.; Orawa, H.; Budach, V.; Jordan, A. Efficacy and Safety of Intratumoral Thermotherapy Using Magnetic Iron-Oxide Nanoparticles Combined with External Beam Radiotherapy on Patients with Recurrent Glioblastoma Multiforme. J. Neurooncol. 2011, 103 (2), 317324.

(84) Xu, H.-L.; Mao, K.-L.; Huang, Y.-P.; Yang, J.-J.; Xu, J.; Chen, P.-P.; Fan, Z.-L.; Zou, S.; Gao, Z.-Z.; Yin, J.-Y.; Xiao, J.; Lu, C.-T.; Zhang, B.-L.; Zhao, Y.-Z. GliomaTargeted Superparamagnetic Iron Oxide Nanoparticles as Drug-Carrying Vehicles for Theranostic Effects. Nanoscale 2016, 8 (29), 14222-14236.

(85) Yin, P. T.; Shah, B. P.; Lee, K.-B. Combined Magnetic Nanoparticle-Based MicroRNA and Hyperthermia Therapy to Enhance Apoptosis in Brain Cancer Cells. Small 2014, 10 (20), 4106-4112.

(86) DeAngelis, L. M. Chemotherapy for Brain Tumors - A New Beginning. N. Engl. J. Med. 2005, 352 (10), 1036-1038.

(87) Chertok, B.; David, A. E.; Yang, V. C. Magnetically-Enabled and MR-Monitored Selective Brain Tumor Protein Delivery in Rats via Magnetic Nanocarriers. 
Biomaterials 2011, 32 (26), 6245-6253.

(88) Muroski, M. E.; Morshed, R. A.; Cheng, Y.; Vemulkar, T.; Mansell, R.; Han, Y.; Zhang, L.; Aboody, K. S.; Cowburn, R. P.; Lesniak, M. S. Controlled Payload Release by Magnetic Field Triggered Neural Stem Cell Destruction for Malignant Glioma Treatment. PLoS One 2016, 11 (1), e0145129.

(89) Marie, H.; Lemaire, L.; Franconi, F.; Lajnef, S.; Frapart, Y.-M.; Nicolas, V.; Frébourg, G.; Trichet, M.; Ménager, C.; Lesieur, S. Superparamagnetic Liposomes for MRI Monitoring and External Magnetic Field-Induced Selective Targeting of Malignant Brain Tumors. Adv. Funct. Mater. 2015, 25 (8), 1258-1269.

(90) Alphandéry, E.; Idbaih, A.; Adam, C.; Delattre, J.-Y.; Schmitt, C.; Guyot, F.; Chebbi, I. Development of Non-Pyrogenic Magnetosome Minerals Coated with Poly-l-Lysine Leading to Full Disappearance of Intracranial U87-Luc Glioblastoma in 100\% of Treated Mice Using Magnetic Hyperthermia. Biomaterials 2017, 141, 210-222.

(91) Weinberg, I.; Benlloch Baviera, J. M. Platform for Image-Guided Non-Invasive Brain Delivery of Magnetic Particles. Concept Design and Technical Progress. IEEE Magn. Lett. 2018, 1-1.

(92) Alphandéry, E.; Idbaih, A.; Adam, C.; Delattre, J.-Y.; Schmitt, C.; Guyot, F.; Chebbi, I. Chains of Magnetosomes with Controlled Endotoxin Release and Partial Tumor Occupation Induce Full Destruction of Intracranial U87-Luc Glioma in Mice under the Application of an Alternating Magnetic Field. J. Control. Release 2017, 262, 259-272.

(93) Mertz, D.; Sandre, O.; Bégin-Colin, S. Drug Releasing Nanoplatforms Activated by Alternating Magnetic Fields. Biochim. Biophys. Acta - Gen. Subj. 2017, 1861 (6), 1617-1641.

(94) Lin, Y.-J.; Chen, K.-T.; Huang, C.-Y.; Wei, K.-C. Non-Invasive Focused UltrasoundBased Synergistic Treatment of Brain Tumors. J. Cancer Res. Pract. 2016, 3 (3), $63-$ 68.

(95) Liu, H.-L.; Hua, M.-Y.; Chen, P.-Y.; Chu, P.-C.; Pan, C.-H.; Yang, H.-W.; Huang, C.Y.; Wang, J.-J.; Yen, T.-C.; Wei, K.-C. Blood-Brain Barrier Disruption with Focused Ultrasound Enhances Delivery of Chemotherapeutic Drugs for Glioblastoma Treatment. Radiology 2010, 255 (2), 415-425.

(96) Yang, F.-Y.; Wang, H.-E.; Liu, R.-S.; Teng, M.-C.; Li, J.-J.; Lu, M.; Wei, M.-C.; Wong, T.-T. Pharmacokinetic Analysis of 111 In-Labeled Liposomal Doxorubicin in Murine Glioblastoma after Blood-Brain Barrier Disruption by Focused Ultrasound. PLoS One 2012, 7 (9), e45468.

(97) Fan, C.-H.; Ting, C.-Y.; Lin, H.-J.; Wang, C.-H.; Liu, H.-L.; Yen, T.-C.; Yeh, C.-K. SPIO-Conjugated, Doxorubicin-Loaded Microbubbles for Concurrent MRI and Focused-Ultrasound Enhanced Brain-Tumor Drug Delivery. Biomaterials 2013, 34 (14), 3706-3715.

(98) Treat, L. H.; McDannold, N.; Vykhodtseva, N.; Zhang, Y.; Tam, K.; Hynynen, K. Targeted Delivery of Doxorubicin to the Rat Brain at Therapeutic Levels Using MRIGuided Focused Ultrasound. Int. J. Cancer 2007, 121 (4), 901-907.

(99) Timbie, K. F.; Afzal, U.; Date, A.; Zhang, C.; Song, J.; Wilson Miller, G.; Suk, J. S.; Price, R. J. MR Image-Guided Delivery of Cisplatin-Loaded Brain-Penetrating Nanoparticles to Invasive Glioma with Focused Ultrasound. J. Control. Release 2017, $263,120-131$.

(100) Lammers, T.; Koczera, P.; Fokong, S.; Gremse, F.; Ehling, J.; Vogt, M.; Pich, A.; Storm, G.; van Zandvoort, M.; Kiessling, F. Theranostic USPIO-Loaded Microbubbles for Mediating and Monitoring Blood-Brain Barrier Permeation. Adv. Funct. Mater. 2015, 25 (1), 36-43.

(101) Aryal, M.; Park, J.; Vykhodtseva, N.; Zhang, Y.-Z.; McDannold, N. Enhancement in 
Blood-Tumor Barrier Permeability and Delivery of Liposomal Doxorubicin Using Focused Ultrasound and Microbubbles: Evaluation during Tumor Progression in a Rat Glioma Model. Phys. Med. Biol. 2015, 60 (6), 2511-2527.

(102) Baghirov, H.; Snipstad, S.; Sulheim, E.; Berg, S.; Hansen, R.; Thorsen, F.; Mørch, Y.; Davies, C. de L.; Åslund, A. K. O. Ultrasound-Mediated Delivery and Distribution of Polymeric Nanoparticles in the Normal Brain Parenchyma of a Metastatic Brain Tumour Model. PLoS One 2018, 13 (1), e0191102.

(103) Yang, H.-W.; Hua, M.-Y.; Hwang, T.-L.; Lin, K.-J.; Huang, C.-Y.; Tsai, R.-Y.; Ma, C.-C. M.; Hsu, P.-H.; Wey, S.-P.; Hsu, P.-W.; Chen, P.-Y.; Huang, Y.-C.; Lu, Y.-J.; Yen, T.-C.; Feng, L.-Y.; Lin, C.-W.; Liu, H.-L.; Wei, K.-C. Non-Invasive Synergistic Treatment of Brain Tumors by Targeted Chemotherapeutic Delivery and Amplified Focused Ultrasound-Hyperthermia Using Magnetic Nanographene Oxide. Adv. Mater. 2013, 25 (26), 3605-3611.

(104) Shibaguchi, H.; Tsuru, H.; Kuroki, M.; Kuroki, M. Sonodynamic Cancer Therapy: A Non-Invasive and Repeatable Approach Using Low-Intensity Ultrasound with a Sonosensitizer. Anticancer Res. 2011, 31 (7), 2425-2429.

(105) Canavese, G.; Ancona, A.; Racca, L.; Canta, M.; Dumontel, B.; Barbaresco, F.; Limongi, T.; Cauda, V. Nanoparticle-Assisted Ultrasound: A Special Focus on Sonodynamic Therapy against Cancer. Chem. Eng. J. 2018, 340, 155-172.

(106) Endo, S.; Kudo, N.; Yamaguchi, S.; Sumiyoshi, K.; Motegi, H.; Kobayashi, H.; Terasaka, S.; Houkin, K. Porphyrin Derivatives-Mediated Sonodynamic Therapy for Malignant Gliomas In Vitro. Ultrasound Med. Biol. 2015, 41 (9), 2458-2465.

(107) Miyoshi, N.; Kundu, S. K.; Tuziuti, T.; Yasui, K.; Shimada, I.; Ito, Y. Combination of Sonodynamic and Photodynamic Therapy against Cancer Would Be Effective through Using a Regulated Size of Nanoparticles. Nanosci. Nanoeng. 2016, 4 (1), 1-11.

(108) Huang, J.; Liu, F.; Han, X.; Zhang, L.; Hu, Z.; Jiang, Q.; Wang, Z.; Ran, H.; Wang, D.; Li, P. Nanosonosensitizers for Highly Efficient Sonodynamic Cancer Theranostics. Theranostics 2018, 8 (22), 6178-6194.

(109) Bogdan, J.; Pławińska-Czarnak, J.; Zarzyńska, J. Nanoparticles of Titanium and Zinc Oxides as Novel Agents in Tumor Treatment: A Review. Nanoscale Res. Lett. 2017, $12(1), 225$.

(110) Chen, M.; Xu, A.; He, W.; Ma, W.; Shen, S. Ultrasound Triggered Drug Delivery for Mitochondria Targeted Sonodynamic Therapy. J. Drug Deliv. Sci. Technol. 2017, 39, 501-507.

(111) Sun, Y.; Wang, H.; Wang, P.; Zhang, K.; Geng, X.; Liu, Q.; Wang, X. Tumor Targeting DVDMS-Nanoliposomes for an Enhanced Sonodynamic Therapy of Gliomas. Biomater. Sci. 2019.

(112) Ultrasound and Nanobots — a Deadly Duo against Cancer. Nature.

(113) Trendowski, M. Using the Promise of Sonodynamic Therapy in the Clinical Setting against Disseminated Cancers. Chemother. Res. Pract. 2015, 2015, 1-16.

(114) Yamamoto, S.; Ono, M.; Yuba, E.; Harada, A. In Vitro Sonodynamic Therapeutic Effect of Polyion Complex Micelles Incorporating Titanium Dioxide Nanoparticles. Nanomater. (Basel, Switzerland) 2017, 7 (9), 16.

(115) Guduru, R.; Khizroev, S. Magnetic Field-Controlled Release of Paclitaxel Drug from Functionalized Magnetoelectric Nanoparticles. Part. Part. Syst. Charact. 2014, 31 (5), 605-611.

(116) Yue, K.; Guduru, R.; Hong, J.; Liang, P.; Nair, M.; Khizroev, S. Magneto-Electric Nano-Particles for Non-Invasive Brain Stimulation. PLoS One 2012, 7 (9), e44040.

(117) Stewart, T. S.; Nagesetti, A.; Guduru, R.; Liang, P.; Stimphil, E.; Hadjikhani, A.; Salgueiro, L.; Horstmyer, J.; Cai, R.; Schally, A.; Khizroev, S. Magnetoelectric 
Nanoparticles for Delivery of Antitumor Peptides into Glioblastoma Cells by Magnetic Fields. Nanomedicine 2018, 13 (4), 423-438.

(118) Peiris, P. M.; Abramowski, A.; Mcginnity, J.; Doolittle, E.; Toy, R.; Gopalakrishnan, R.; Shah, S.; Bauer, L.; Ghaghada, K. B.; Hoimes, C.; Brady-Kalnay, S. M.; Basilion, J. P.; Griswold, M. A.; Karathanasis, E. Treatment of Invasive Brain Tumors Using a Chain-like Nanoparticle. Cancer Res. 2015, 75 (7), 1356-1365.

(119) Karathanasis, E.; Ghaghada, K. B. Crossing the Barrier: Treatment of Brain Tumors Using Nanochain Particles. Wiley Interdiscip. Rev. Nanomedicine Nanobiotechnology 2016, 8 (5), 678-695.

(120) Kaushik, A.; Jayant, R. D.; Sagar, V.; Nair, M. The Potential of Magneto-Electric Nanocarriers for Drug Delivery. Expert Opin. Drug Deliv. 2014, 11 (10), 1635-1646.

(121) Stimphil, E.; Nagesetti, A.; Guduru, R.; Stewart, T.; Rodzinski, A.; Liang, P.; Khizroev, S. Physics Considerations in Targeted Anticancer Drug Delivery by Magnetoelectric Nanoparticles. Appl. Phys. Rev. 2017, 4 (2), 021101.

(122) Gao, H. Perspectives on Dual Targeting Delivery Systems for Brain Tumors. $J$. Neuroimmune Pharmacol. 2017, 12 (1), 6-16.

(123) Liu, W.; Miroshnichenko, A. E.; Neshev, D. N.; Kivshar, Y. S. Broadband Unidirectional Scattering by Magneto-Electric Core-Shell Nanoparticles. ACS Nano 2012, 6 (6), 5489-5497.

(124) Nair, M.; Guduru, R.; Liang, P.; Hong, J.; Sagar, V.; Khizroev, S. Externally Controlled On-Demand Release of Anti-HIV Drug Using Magneto-Electric Nanoparticles as Carriers. Nat. Commun. 2013, 4 (1), 1707.

(125) Saenz del Burgo, L.; Hernández, R. M.; Orive, G.; Pedraz, J. L. Nanotherapeutic Approaches for Brain Cancer Management. Nanomedicine Nanotechnology, Biol. Med. 2014, 10 (5), e905-e919.

(126) Lei, C.; Davoodi, P.; Zhan, W.; Chow, P. K.-H.; Wang, C.-H. Development of Nanoparticles for Drug Delivery to Brain Tumor: The Effect of Surface Materials on Penetration Into Brain Tissue. J. Pharm. Sci. 2018.

(127) Dong, X. Current Strategies for Brain Drug Delivery. Theranostics 2018, 8 (6), 14811493.

(128) Thomsen, L. B.; Thomsen, M. S.; Moos, T. Targeted Drug Delivery to the Brain Using Magnetic Nanoparticles. Ther. Deliv. 2015, 6 (10), 1145-1155.

(129) D’Agata, F.; Ruffinatti, F.; Boschi, S.; Stura, I.; Rainero, I.; Abollino, O.; Cavalli, R.; Guiot, C.; D’Agata, F.; Ruffinatti, F. A.; Boschi, S.; Stura, I.; Rainero, I.; Abollino, O.; Cavalli, R.; Guiot, C. Magnetic Nanoparticles in the Central Nervous System: Targeting Principles, Applications and Safety Issues. Molecules 2017, 23 (1), 9.

(130) Hoff, D.; Sheikh, L.; Bhattacharya, S.; Nayar, S.; Webster, T. J. Comparison Study of Ferrofluid and Powder Iron Oxide Nanoparticle Permeability across the Blood-Brain Barrier. Int. J. Nanomedicine 2013, 8, 703-710.

(131) Cintra e Silva, D. de O.; Estevanato, L. L. C.; Simioni, A. R.; de Andrade Rodrigues, M. M.; Lacava, B. M.; Lacava, Z. G. M.; Tedesco, A. C.; Morais, P. C.; Báo, S. N. Successful Strategy for Targeting the Central Nervous System Using Magnetic Albumin Nanospheres. J. Biomed. Nanotechnol. 2012, 8 (1), 182-189.

(132) Luo, Z.; Jin, K.; Pang, Q.; Shen, S.; Yan, Z.; Jiang, T.; Zhu, X.; Yu, L.; Pang, Z.; Jiang, X. On-Demand Drug Release from Dual-Targeting Small Nanoparticles Triggered by High-Intensity Focused Ultrasound Enhanced Glioblastoma-Targeting Therapy. ACS Appl. Mater. Interfaces 2017, 9 (37), 31612-31625.

(133) Min, H. S.; You, D. G.; Son, S.; Jeon, S.; Park, J. H.; Lee, S.; Kwon, I. C.; Kim, K. Echogenic Glycol Chitosan Nanoparticles for Ultrasound-Triggered Cancer Theranostics. Theranostics 2015, 5 (12), 1402-1418. 
(134) Cheng, Y.; Morshed, R. A.; Auffinger, B.; Tobias, A. L.; Lesniak, M. S. Multifunctional Nanoparticles for Brain Tumor Imaging and Therapy. Adv. Drug Deliv. Rev. 2014, 66, 42-57.

(135) Hu, C.; Liang, K.; An, R.; Wang, X.; You, L. The Characterization, Pharmacokinetic, and Tissue Distribution Studies of TPGS-Modified Artesunate Liposome in Rats. Drug Dev. Ind. Pharm. 2018, 44 (9), 1528-1535.

(136) Cole, A. J.; David, A. E.; Wang, J.; Galbán, C. J.; Yang, V. C. Magnetic Brain Tumor Targeting and Biodistribution of Long-Circulating PEG-Modified, Cross-Linked Starch-Coated Iron Oxide Nanoparticles. Biomaterials 2011, 32 (26), 6291-6301.

(137) Qiu, Y.; Park, K. Environment-Sensitive Hydrogels for Drug Delivery. Adv. Drug Deliv. Rev. 2001, 53 (3), 321-339.

(138) Karabeber, H.; Huang, R.; Iacono, P.; Samii, J. M.; Pitter, K.; Holland, E. C.; Kircher, M. F. Guiding Brain Tumor Resection Using Surface-Enhanced Raman Scattering Nanoparticles and a Hand-Held Raman Scanner. ACS Nano 2014, 8 (10), 9755-9766.

(139) van Tellingen, O.; Yetkin-Arik, B.; de Gooijer, M. C.; Wesseling, P.; Wurdinger, T.; de Vries, H. E. Overcoming the Blood-brain Tumor Barrier for Effective Glioblastoma Treatment. Drug Resist. Updat. 2015, 19, 1-12.

(140) Drew, L. Pharmacogenetics: The Right Drug for You. Nature 2016, 537 (7619), S60S62.

(141) Rivnay, J.; Wang, H.; Fenno, L.; Deisseroth, K.; Malliaras, G. G. Next-Generation Probes, Particles, and Proteins for Neural Interfacing. Sci. Adv. 2017, 3 (6), e1601649. 